\title{
Identification of the SHREK family of proteins as broad-spectrum host antiviral factors
}

Deemah Dabbagh, Sijia He, Brian Hetrick, Linda Chilin, Ali Andalibi, and Yuntao Wu*

National Center for Biodefense and Infectious Diseases, School of Systems Biology, George Mason University, Manassas, VA 20110, USA.

*Correspondence: ywu8@gmu.edu

Keywords: SHREK, PSGL-1, CD34, PODXL1, PODXL2, CD164, MUC4, HIV-1, Influenza A, SARS-CoV-2 


\begin{abstract}
Mucins and mucin-like molecules are highly glycosylated, high-molecular-weight cell surface proteins that possess a semi-rigid and highly extended extracellular domain. P-selectin glycoprotein ligand-1 (PSGL-1), a mucin-like glycoprotein, has recently been found to restrict HIV-1 infectivity through virion incorporation that sterically hinders virus particle attachment to target cells. Here, we report the identification of a family of antiviral cellular proteins, named the Surface-Hinged, Rigidly-Extended Killer (SHREK) family of virion inactivators (PSGL-1,

CD43, TIM-1, CD34, PODXL1, PODXL2, CD164, MUC1, MUC4, and TMEM123), that share similar structural characteristics with PSGL-1. We demonstrate that SHREK proteins block HIV1 infectivity by inhibiting virus particle attachment to target cells. In addition, we demonstrate that SHREK proteins are broad-spectrum host antiviral factors that block the infection of diverse viruses such as influenza A. Furthermore, we demonstrate that a subset of SHREKs also blocks the infectivity of a hybrid alphavirus-SARS-CoV-2 virus-like particle. These results suggest that SHREK proteins may be a part of host innate immunity against enveloped viruses.
\end{abstract}




\section{INTRODUCTION}

Mucins and mucin-like molecules are highly glycosylated, high-molecular-weight cell surface proteins that possess a semi-rigid and highly extended extracellular domain (Hattrup and Gendler, 2008). P-selectin glycoprotein ligand-1 (PSGL-1), a mucin-like glycoprotein (Goetz et al., 1997; Guyer et al., 1996; Sako et al., 1993) has recently been found to restrict HIV-1 infectivity (Liu et al., 2019) through steric hindrance (Fu et al., 2020; Murakami et al., 2020); mechanistically, PSGL-1 was found to be incorporated onto virion particles, which inhibits virion attachment to target cells (Fu et al., 2020; Murakami et al., 2020). The extracellular domain of PSGL-1 was found to be necessary for its antiviral activity (Fu et al., 2020). PSGL-1 shares structural features with other mucins and mucin-like proteins; it has a heavily glycosylated, elongated extracellular domain that extends nearly $60 \mathrm{~nm}$ from the plasma membrane (Li et al., 1996a; Li et al., 1996b; McEver et al., 1995; Patel et al., 1995) and sterically hinders virus attachment to target cells when incorporated into virions (Fu et al., 2020; Murakami et al., 2020).

To test whether molecules with a similar structure would also block HIV-1 virion infectivity, we selected a group of cellular proteins, including CD43, TIM-1, CD34, PODXL1, PODXL2, CD164, MUC1, MUC4, and TMEM123. These proteins have diverse coding sequences, tissue expression patterns, and functionalities, but share a similar structural feature, namely a highly extended and heavily glycosylated extracellular domain. CD43 is a sialomucin transmembrane protein expressed at high levels on T lymphocytes, monocytes, and some B lymphocytes (Pallant et al., 1989). TIM-1 is preferentially expressed on Th2 T cells, and also contains an extracellular mucin domain and an N-terminal immunoglobulin (Ig)-like domain (Umetsu et al., 2005). Both proteins have previously been shown to block HIV-1 infection of T cells (Fu et al., 2020; Li et al., 2019; Murakami et al., 2020). CD34, the common surface marker of stem and progenitor cells (Sidney et al., 2014), is also a member of the sialomucin family of proteins, which contain an extensively $\mathrm{O}$-glycosylated extracellular mucin-like domain. The podocalyxin-like proteins 1 and 2 (PODXL1 and PODXL2) are CD34-related sialomucins and share a similar structure with CD34 (Nielsen and McNagny, 2008), and are also makers of hematopoietic stem and progenitor cells (Doyonnas et al., 2005; Sassetti et al., 2000). CD164 is another sialomucin co-expressed with CD34 on the surface of human hematopoietic cells, and is structurally similar to CD34 (Watt and Chan, 2000). We also selected two mucins, MUC1 and MUC4, which are transmembrane mucins with dense arrays of O-linked oligosaccharides attached to the threonine and serine residues in the extracellular domains (Hattrup and Gendler, 2008). TMEM123 (Porimin) is another mucin-like protein with high contents of glycosylated threonine and serine residues in its extracellular domain (Ma et al., 2001).

In this study, we tested these mucins and mucin-like proteins for their ability to inactivate HIV-1 infectivity, and found that all these proteins block the infectivity of HIV-1 through inhibiting virus particle attachment to target cells. In addition, we also demonstrated that SHREK proteins are broad-spectrum host antiviral factors that block the infection of diverse viruses such as influenza A. These results suggest that SHREK proteins may be a part of host innate immunity against enveloped viruses. 


\section{RESULTS}

\section{Inactivation of HIV-1 virion infectivity by mucins and mucin-like proteins}

Previous studies have demonstrated that deletion of the highly glycosylated extracellular domain of PSGL-1 abolished its antiviral activity (Fu et al., 2020). The extracellular domain of PSGL-1 consists of 14-16 decameric repeats (DR) with multiple $O$-glycosylated threonines (30\%) and prolines (10\%) (Baisse et al., 2007; Tauxe et al., 2008). DR plays a pivotal role in elongating and strengthening the extracellular N-terminal domain (Tauxe et al., 2008). We found that when the DR domain was deleted from the N-terminus, the anti-HIV activity of PSGL-1 was also abolished (Figure 1A and 1C) (Fu et al., 2020). These results demonstrate that the heavily glycosylated, large extracellular domain of PSLG-1 plays a critical role in blocking virion infectivity through steric hindrance (Fu et al., 2020; Murakami et al., 2020). To determine whether mucins and mucin-like proteins with a similar extracellular structure would also inhibit HIV-1 virion infectivity, we selected and tested a group of mucins and mucin-like proteins, including CD43, TIM-1, CD34, PODXL1, PODXL2, CD164, MUC1, MUC4, and TMEM123 (Figure 1A). We cotransfected them individually with HIV-1(NL4-3) DNA into HEK293T cells to assemble viral particles, and then quantified the infectivity of the virions by infecting an HIV1 Rev-dependent indicator cell, Rev-A3R5-GFP (Fu et al., 2020; Wu et al., 2007a, b) (using an equal level of $\mathrm{p} 24)$. The presence of each of these proteins in virus producer cells blocked the infectivity of HIV-1 virions released (Figure 1D).

To determine whether transmembrane proteins with large extracellular domains would similarly inhibit HIV-1 infectivity, we selected several non-mucin proteins with various extracellular repeat domains. Both CD2 and ICAM-1 are members of the immunoglobulin (Ig) superfamily of proteins that contain heavily glycosylated extracellular Ig domains ( 2 and $5 \mathrm{Ig}$ domain repeats for CD2 and ICAM-1, respectively) (Barclay, 2003). L-selectin and E-selectin are selectin family of cell adhesion molecules that share a similar extracellular structure with a variable number of consensus repeats ( 2 and 6 for L- and E-, respectively) (Ley, 2003). We also selected integrin beta chain-2 (IGTB2), which has a large extracellular domain with integrin epidermal growth factor like repeat domains (I-EGF repeats 1 to 4) (Hynes, 2002). Each of these proteins was similarly cotransfected with HIV-1(NL4-3) DNA into HEK293T cells to assemble viral particles. Virions released were harvested and quantified for infectivity in Rev-A3R5-GFP cells (using an equal level of p24). As shown in Figure 1E, none of these proteins, except for E-selectin (Figure 1 Supplement 1), was found to block the infectivity of HIV-1 virions. This is in contrast with the mucin and mucin-like proteins that we selected; all of these mucin and mucin-like proteins were found to block HIV-1 infectivity (Figure 1D). These results demonstrate that the ability of mucin and mucin-like proteins to block HIV-1 infectivity is not a shared property of transmembrane proteins expressed in virus producer cells.

We further performed experiments to quantify and compare the anti-HIV activity of the mucin and mucin-like proteins. We observed dosage-dependent inhibition of HIV-1 by all of them (Figure 2A, 2B, and Figure 2 Supplement 1 to Figure 2 Supplement 7), and the 50\% inhibition dosages were determined (Figure 2C). Among these proteins, CD34, the common stem cell maker, had the most potent anti-HIV activity, blocking HIV-1 virion infectivity at an IC50 of $2.33 \mathrm{ng}$ (Figure 2C); MUC1 was the least potent, with an IC50 of $216.06 \mathrm{ng}$ (Figure 2C). Based on the antiviral activities and shared structural feature of these proteins, we conveniently named them the Surface-Hinged, Rigidly-Extended Killer (SHREK) family of virion inactivators. 


\section{Effects of SHREK protein expression on HIV-1 virion release}

To elucidate the anti-HIV mechanisms of SHREK proteins, we first examine the effects of SHREK expression on virion release in virus producer cells. Previous studies of PSGL-1 has demonstrated that expression of PSGL-1 in virus producer cells had minimal effects on HIV-1 virion release (Fu et al., 2020). To examine effects of SHREK proteins on HIV-1 virion release, we co-transfected HEK293T cells with HIV(NL4-3) proviral DNA $(1 \mu \mathrm{g})$ plus each of the SHREK protein expression vector at varying inputs from 0.5 to $400 \mathrm{ng}$ (Figure 3). The release of virions in the presence of each SHREK protein was quantified. All of the proteins except TIM-1 had minor effects on virion release at doses below $100 \mathrm{ng}$ (Figure 3). CD34, PODXL1, and CD164 (McLaren et al., 2015) inhibited HIV-1 virion release only at high dosages (100 ng and above). TIM-1 potently inhibited virion release at doses as low as 5-10 ng, consistent with a previous report (Li et al., 2019). While not inhibiting virion release at low dosages, all of these proteins, except for MUC1 (IC50, 216.05), effectively blocked virion infectivity with an IC50 at approximately $10 \mathrm{ng}$ and below (Figure 2B and 2C). These results suggested that similar to PSGL-1, inactivating virion infectivity rather than blocking virion release is a major mechanism of SHREK proteins.

\section{Virion incorporation of SHREK proteins and effects on HIV-1 Env incorporation}

The anti-HIV activity of PSGL-1 is mainly attributed to its incorporation into virion particles that sterically hinders virion attachment to target cells (Fu et al., 2020; Murakami et al., 2020). In addition, virion incorporation of PSGL-1 also inhibits the incorporation of the HIV-1 Env protein (Fu et al., 2020; Liu et al., 2020). To study the anti-viral mechanisms of SHREK, we examined virion incorporation of SHERK proteins. For this purpose, we adopted a previously established particle pull-down assay (Thibault et al., 2007), using magnetic beads-conjugated anti-SHREK antibodies to pull down virion particles that express SHREK on the surface (Figure 4A).

Magnetically purified virions were quantified for the amounts of HIV-1 p24 in the virions. We were able to demonstrate that the anti-PSGL-1, -CD43, -CD164, -PODXL1, -PODXL2, and CD34 antibodies selectively pulled down virion particles produced from these SHREK expression cells, demonstrating virion incorporation of SHREK proteins. The anti-TMEM123 antibody had a weak ability to pull down virion particles produced from the TMEM123 expression cells (Figure 4A). We were not able to use the commercial anti-MUC1 and antiMUC4 antibodies to pull down virion particles.

We further examined effects of SHREK incorporation on HIV-1 Env incorporation. Virion particles were assembled in the presence or absence of SHREK proteins, and then analyzed by western blots for HIV-1 Env gp41 and p24. As shown in Figure 4C, all SHREKs, except for TMEM123, had minor inhibition of HIV-1 Env incorporation to various degrees; while TMEM123 did not affect Env incorporation, PODXL2 had the strongest inhibition.

\section{SHREK proteins inhibit virus particle attachment to target cells}

We and others previously reported that PSGL-1-mediated inhibition of virion infectivity occurs mainly through virion incorporation, leading to PSGL-1-mediated steric hindrance of particle attachment to target cells (Fu et al., 2020; Murakami et al., 2020). We performed a similar virion 
attachment assay using particles produced from cells expressing each individual SHREK protein. As shown in Figure 5A, as a control, particles produced from the PSGL-1-expressing cells were highly impaired in their ability to attach to target cells. Similar strong impairment was observed for virions produced from the CD34- and the PODXL2-expressing cells. Of notes, these three proteins (PSGL-1, CD34, and PODXL2) are also the most potent virion inactivators among SHREKs, with an IC50 around 2 ng (Figure 2C). The other proteins (CD43, CD164, PODXL1, MUC1, and MUC4) also inhibited virion attachment to varying degrees. Interestingly, TMEM123 slightly enhanced HIV-1 virion attachment to target cells, although it inhibited viral infection (Figure 2B and 2C). One possible reason is that TMEM123 itself may interact with surface proteins, thereby promoting non-productive attachment of virus particles to target cells. Indeed, when the binding assay was performed in the presence of an anti-TMEM123 antibody to block possible TMEM123 interaction with cell surface proteins, the enhancement of viral binding was diminished, and virion attachment was decreased (Figure 5B).

\section{SHREK proteins are broad-spectrum host antiviral factors}

PSGL-1 has been shown to be a broad-spectrum host antiviral factors (Fu et al., 2020). To further determine whether these SHREK proteins also possess broad-spectrum antiviral activities, we tested their ability to block other viruses, including influenza A and SARS-CoV-2 virus-like particles (VLP). To assemble influenza A virus, eight vectors expressing each of the segments of the influenza A/WSN/33 (H1N1) genome plus a GFP-reporter vector were cotransfected with individual SHREK proteins into HEK293T (Figure 6A). Viral particles were harvested and used to infect target MDCK cells. As shown in Figure 6B, the presence of each of the SHREK proteins in virus-producer cells inhibited the infection of target cells by the virions released. However, the degree of inhibition was different; MUC1, PODXL1, and MUC4 had the strongest inhibition of influenza A virus, although MUC1 and MUC4 were less effective against HIV-1. CD164 and TMEM123 had the weakest inhibition of influenza A. These results demonstrate that SHREK proteins are broad-spectrum and can block the infectivity of multiple viruses. Nevertheless, it is apparent that for each individual SHREK, its antiviral potency can vary among different viruses, with the differences likely related to possible viral antagonisms, the localization of SHREKs and sites of viral budding, and other unidentified factors.

To test possible effects of SHREK on SARS-CoV-2 infection, we assembled a newly developed hybrid alphavirus-SARS-CoV-2 particles (Ha-CoV-2) (Hetrick et al., 2020) in the presence of individual SHREKs, using PSGL-1 as a control. Ha-CoV-2 is a non-replicating SARS-CoV-2 virus-like particle, composed of only SARS-CoV-2 structural proteins (S, M, N, and E) and an RNA genome derived from alphavirus vector. Our recent study has shown that PSGL-1 can block SARS-CoV-2 S protein virion incorporation, virus attachment, and Ha-CoV-2 infection of target cells (He et al., 2021). We performed similar experiments on the inhibition of $\mathrm{Ha}-\mathrm{CoV}-2$ infectivity by SHREKs, and found that in addition to PSGL-1, CD164, TIM-1, MUC1, and MUC4 also inhibited the infection of Ha-CoV-2 virus (Figure 6C); MUC4 had the strongest inhibition among these SHREKs. However, we did not find inhibition of Ha-CoV-2 by CD43, CD34, PODXL1, PODXL2, and TMEM123 (Figure 6 Supplement 1), although they are potent inhibitors of HIV-1 (Figure 2B and 3C). 


\section{DISCUSSION}

In this report, we identified a group of proteins, namely SHREK, that share similar structural characteristics and the ability to broadly inactivate virion infectivity (Figure 1). We also quantified their anti-HIV activity using a range of doses (from $0.5 \mathrm{ng}$ to $400 \mathrm{ng}$ of SHREK expression vector), and observed a strictly dosage-dependent inhibition of HIV-1 virion infectivity (Figure 2). The physiological relevance of SHREK proteins such as PSGL-1 in restricting HIV-1 virion infectivity has been previously confirmed in HIV-1 infection of primary blood CD4 T cells, and by shRNA knockdown of PSGL-1 in human CD4 T cells (Fu et al., 2020); the slight reduction of endogenous levels of PSGL-1 in transformed and primary CD4 T cells led to an enhancement of virion infectivity, demonstrating that the presence of endogenous PSGL-1 can affect virion infectivity (Fu et al., 2020).

Although we mainly focused on proteins with mucin and mucin-like domains, we expect that SHREK proteins will likely include other proteins, such as E-selectin (Figure 1 Supplement 1), with diverse molecular structures. The antiviral activities of SHREK can be achieved through at least 3 different mechanisms: (1) blocking virion release (e.g. TIM-1 inhibition of HIV-1 release (Li et al., 2019)); (2) inhibition of virion incorporation of viral attachment proteins (e.g. PSGL-1 inhibition of HIV-1 gp160 incorporation (Fu et al., 2020)); (3) virion incorporation of SHREK that blocks progeny virus attachment to target cells through steric hindrance (e.g. PSGL-1 inhibition of HIV-1 virus attachment to target cells (Fu et al., 2020; Murakami et al., 2020)). Our previous studies have also shown that viruses such as HIV-1 can evolve antagonisms to counteract the blockage imposed by SHREK proteins. For example, HIV-1 uses the accessory proteins Vpu and Nef to degrade and downregulate PSGL-1 on CD4 T cells (Fu et al., 2020; Liu et al., 2019). However, it remains to be determined what other antagonisms may exist, by which different viruses antagonize individual SHREKs.

Interestingly, we noticed that multiple SHREKs (CD34, PODXL1, PODXL2, and CD164) identified in this study are heavily expressed on the surface of stem and progenitor cells. In particular, CD34, the common surface marker of stem and progenitor cells, is the most potent SHREK against retroviruses such as HIV-1 in our study (Figure 2C). It is likely that these SHREKs are a part of host innate antiviral mechanisms that limit retroviral replication in critical cell populations such as stem and progenitor cells. It has been known that HIV-1 can enter and express genes in CD34+ multipotent hematopoietic progenitor cells (HPCs), but viral replication is limited (Carter et al., 2010). However, viral replication can occur in HPCs with GM-CSF and TNF- $\alpha$ treatment, which induces myeloid differentiation. Such cytokine-induced viral permissiveness coincides with cytokine-mediated CD34 removal from the cell surface (Carter et al., 2010).

We found that several SHREKs (PSGL-1, CD164, TIM-1, MUC1, and MUC4) inhibited HaCoV-2 infection. MUC1 and MUC4 are closely related mucins; MUC4 had the strongest inhibition among these SHREKs in our infection assay. Purified cell-free human breast milk mucins have been shown to possess anti-HIV activity (Habte et al., 2007). A recent study has suggested that MUC4 expression plays a protective role in female mice in SARS-CoV infection (Plante et al., 2020). In addition, in chikungunya virus (CHIKV) infection, the loss of MUC4 also results in augmented disease during early time points, indicating a possible broad role for MUC4 in viral infection and pathogenesis (Plante et al., 2020). 
We found no inhibition of Ha-CoV-2 by CD43, CD34, PODXL1, PODXL2, and TMEM123 (Figure 6 Supplement 1), although these proteins are potent inhibitors of HIV-1 (Figure 2B and $3 C$ ). It is possible that the difference may result from the different sites of viral assembly and budding. SARS-CoV-2 budding occurs mainly at the membranes of ER-Golgi intermediate compartment (V'kovski et al., 2020), whereas HIV-1 buds from the plasma membrane (Freed, 2015). Because of the differences, it is possible that different sets of cellular proteins may be incorporated into HIV-1 and SARS-CoV-2.

Our identification of the SHREK family of proteins offers potential new antiviral therapeutics that may be developed through the induction and modulation of SHREK activities and the inhibition of viral antagonisms. 


\section{ACKNOWLEDGMENTS}

The authors wish to thank the NIH AIDS Reagent Program for reagents. Dr. Feng Li for providing influenza viral expression vectors, Jennifer Guernsey for editorial assistance.

\section{ADDITIONAL INFORMATION}

\section{Funding}

This work was funded in part by US Public Health Service grant 1R01AI148012 from NIAID to Y.W.

\section{Author Contributions}

Experiments were designed by Y.W. Manuscript was written by Y.W. and edited by A.A. Experiments were performed by D.D., S.H., B.H, and L.C.

\section{Competing Interests}

A provisional patent application pertaining to the results presented in this paper has been filed by George Mason University.

\section{Resource Availability}

\section{Lead Contact}

Further information and requests for resources and reagents should be directed to and will be fulfilled by the Lead Contact, Yuntao Wu (ywu8@gmu.edu)

\section{Materials Availability}

This study did not generate new unique reagents.

\section{Data Availability}

The published article includes all data generated or analyzed during this study. 

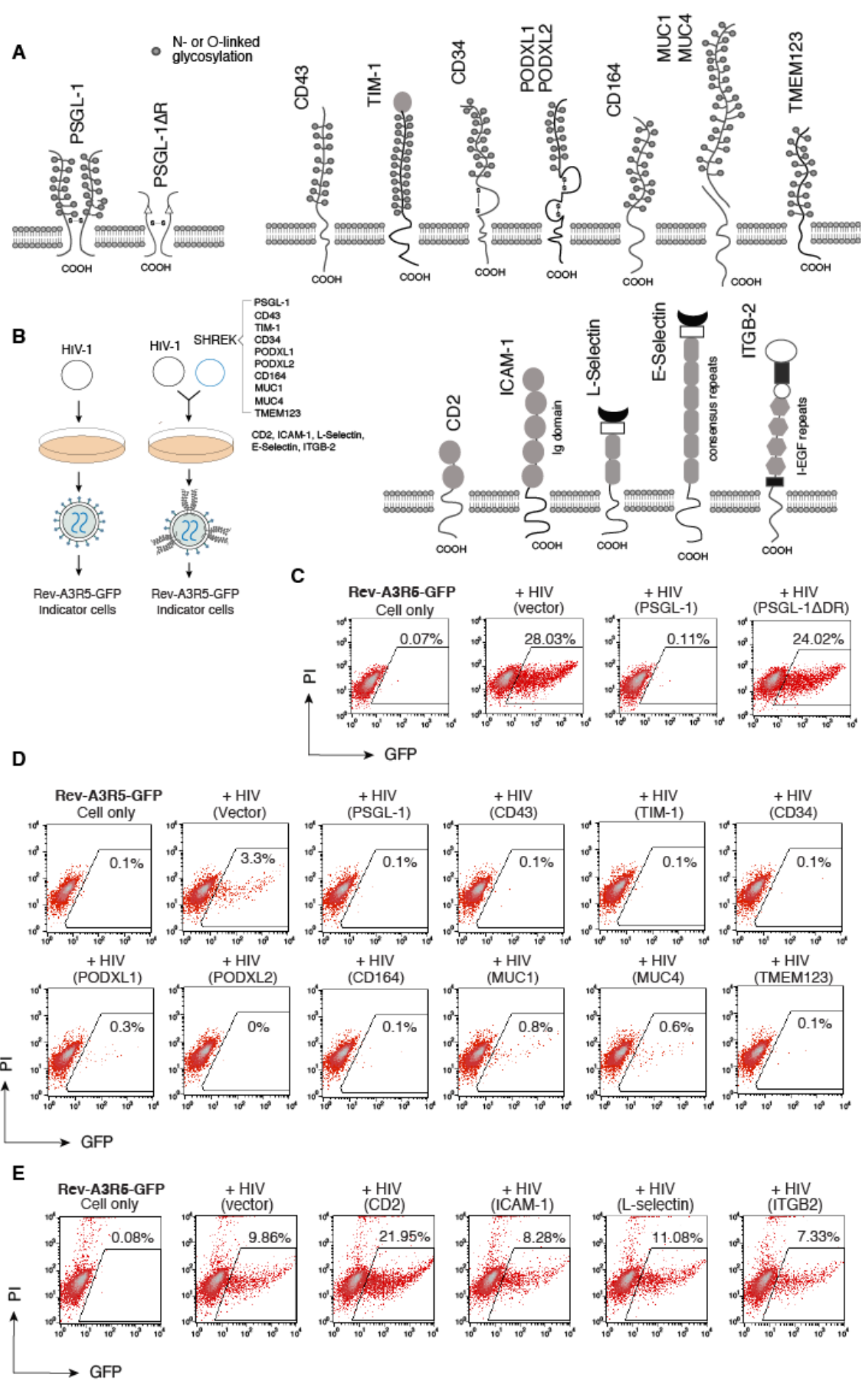

Figure 1. SHREK proteins inactivate HIV-1 virion infectivity.

(A) Schematic of PSGL-1 and PSGL-1 $\triangle$ DR mutant, SHREK proteins, and other surface receptors used in this study. (B) Schematic of virion production in the presence of SHREK proteins in virus producer cells. (C) Requirement of the DR domain of PSGL-1 for blocking HIV-1 infectivity. HEK293T cells were cotransfected with HIV(NL4-3) DNA $(1 \mu \mathrm{g})$ plus the vector expressing PSGL-1 or PSGL-1 $\Delta$ DR (400 
ng). Virions were harvested at 48 hours post-transfection and normalized for $\mathrm{p} 24$, and viral infectivity was quantified by infecting Rev-A3R5-GFP indicator cells. HIV-1 replication was quantified by GFP expression at 72 hours post infection. (D) SHREK proteins inactivate HIV-1 virion infectivity. HEK293T cells were cotransfected with HIV(NL4-3) DNA (1 $\mu \mathrm{g})$ plus each individual SHREK protein (500 ng). Virions were harvested at 48 hours and normalized for $\mathrm{p} 24$, and viral infectivity was quantified by infecting Rev-A3R5-GFP indicator cells. Shown are the percentages of GFP+ cells at 48 hours post infection. (E) For comparison, cells were also similarly cotransfected with HIV-1(NL4-3) DNA plus an empty vector or the vector expressing CD2, ICAM-1, L-selectin, or ITGB2. Virions were harvested and quantified by infecting Rev-A3R5-GFP indicator cells. 
A
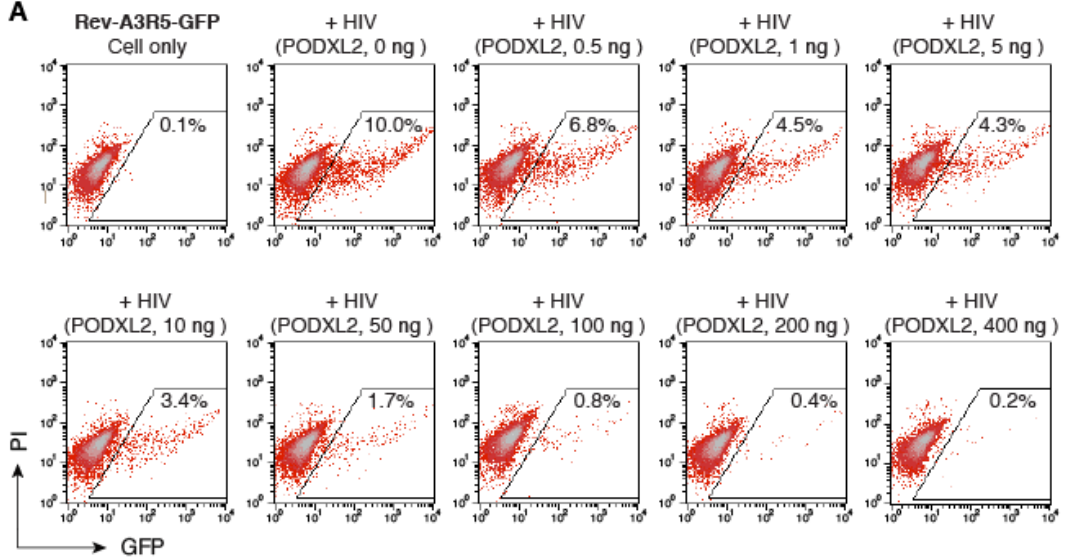

B
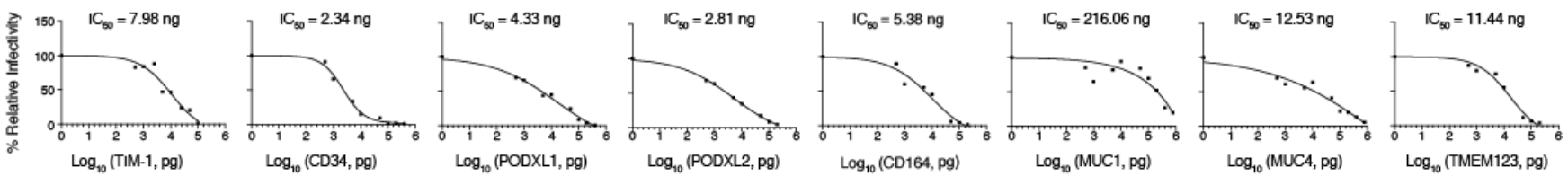

Figure 2. Dosage-dependent inhibition of HIV-1 by SHREK proteins.

(A) Dose-dependent inhibition of HIV-1 infectivity by PODXL2. HEK293T cells were co-transfected with HIV(NL4-3) DNA (1 $\mu \mathrm{g})$ plus the PODXL2 expression vector (0.5 to $400 \mathrm{ng}$ of DNA). Virions were harvested at 48 hours and normalized for $\mathrm{p} 24$, and viral infectivity was quantified by infecting RevA3R5-GFP indicator cells. (B) Virions were assembled in the presence of each individual SHERK protein, and quantified for virus infectivity as in (A). The dose-dependent inhibition of HIV-1 curve by each individual SHREK protein was plotted using results from 3 independent experiments. (C) The 50\% inhibition dosage (IC50) for each SHREK protein was calculated using PRISM software (Graphpad Software Inc.). 

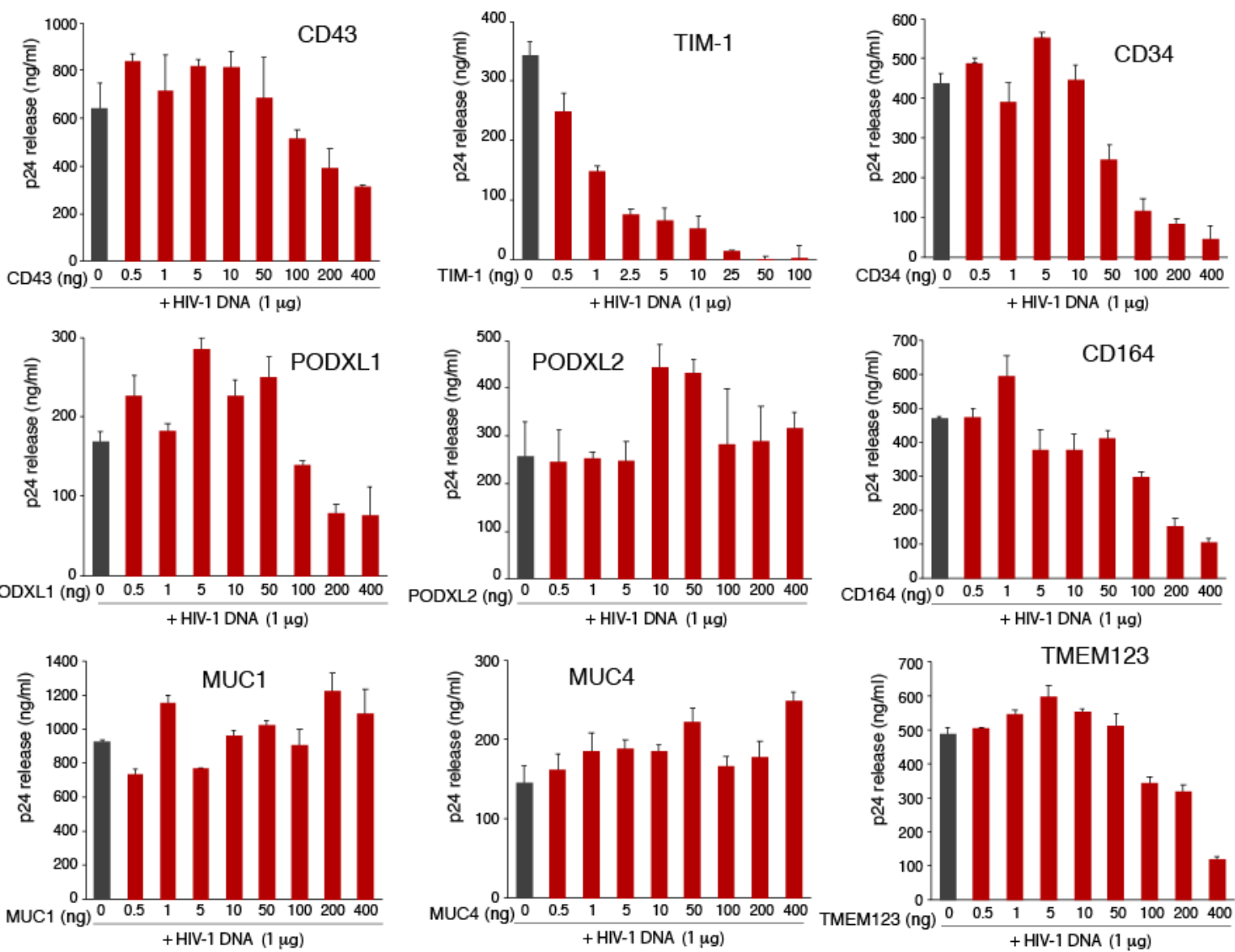

Figure 3. Effects of SHREK proteins on HIV-1 viral release.

HEK293T cells were cotransfected with HIV(NL4-3) DNA $(1 \mu \mathrm{g})$ plus each individual SHREK expression vector ( 0.5 to $400 \mathrm{ng}$ of DNA). Virion release was quantified at 48 hours post cotransfection by HIV-1 p24 ELISA. Data are represented as mean \pm SD from triplicate assays. 
A

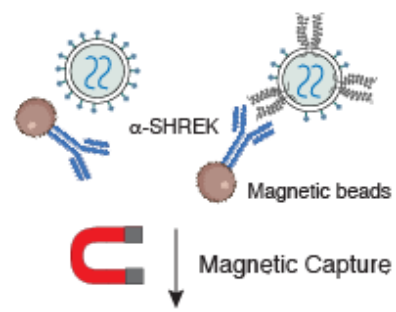

Wash away unbound virus

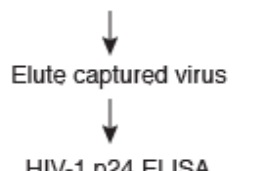

HIV-1 p24 ELISA
B

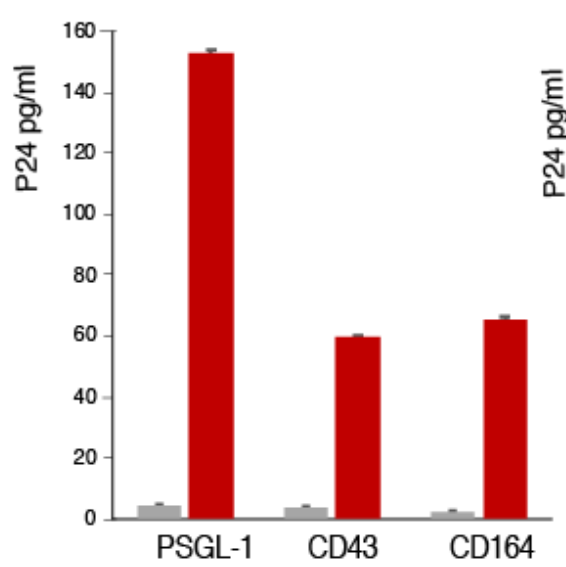

HIV-1+ SHREK

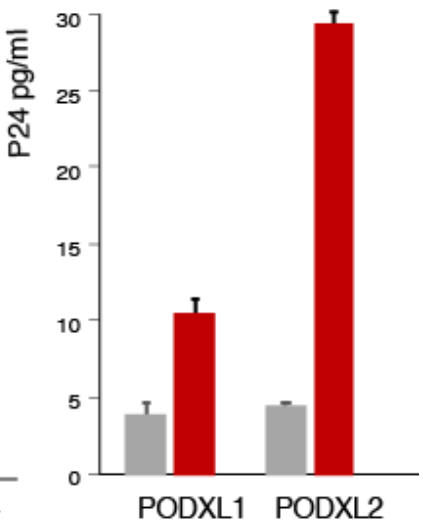

C

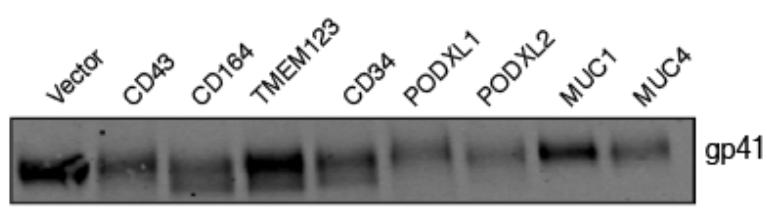
p24

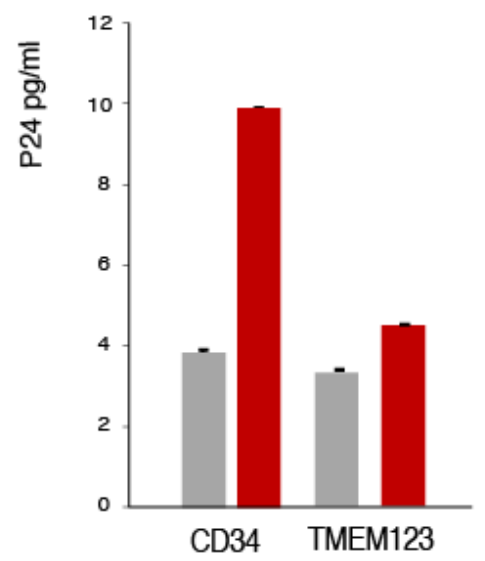

Figure. 4. Virion incorporation of SHREK proteins and its effects on HIV-1 Env incorporation.

(A) Schematic of the immune-magnetic capture assay used to detect SHREK proteins on HIV-1 particles. (B) HEK293T cells were cotransfected with HIV(NL4-3) DNA (1 $\mu \mathrm{g})$ plus each individual SHREK protein expression vector or an empty vector (400 ng of DNA). Particles were harvested at 48 hours and captured with magnetic bead-coated antibodies for each individual SHREK. Captured particles were washed, eluted, and quantified for the p24 levels with HIV-1 p24 ELISA. Data are presented as triplicate means \pm SD. (C) Effect of SHREK proteins on HIV-1 Env incorporation. HEK293T cells were cotransfected with HIV(NL4-3) DNA plus each individual SHREK protein expression vector or an empty vector. Particles were harvested at 48 hours, and purified through a sucrose gradient. Virions were lysed and analyzed with western blot using antibodies against HIV-1 gp41 and p24. Representative blots from 3 repeats are shown. 


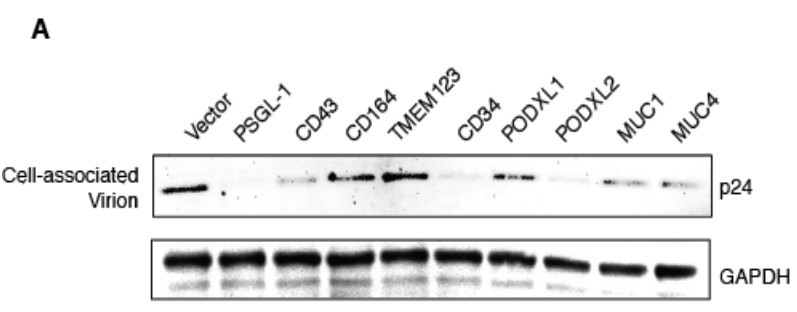

B

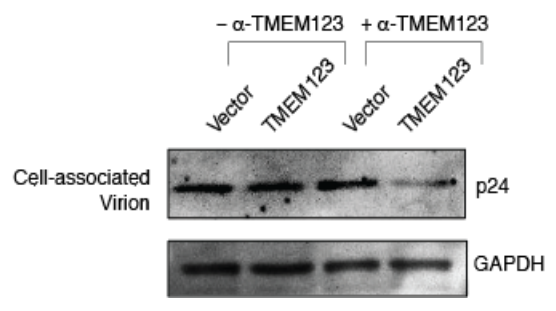

Figure. 5. SHREK proteins block HIV-1 virion attachment to target cells.

(A) Viral particles were produced by cotransfection of HEK293T cells with HIV-1 NL4-3 DNA (1 $\mu \mathrm{g})$ and each individual SHREK protein expression vector or an empty vector (500 ng). HIV-1 p24normalized viral particles were then assayed for attachment to target HeLaJC.53 cells and analyzed by Western blot for cell-bound p24. (B) Virions produced in the presence of the TMEM123 expression vector or the empty vector were assayed for attachment in the presence or absence of an anti-TMEM123 antibody. 
A
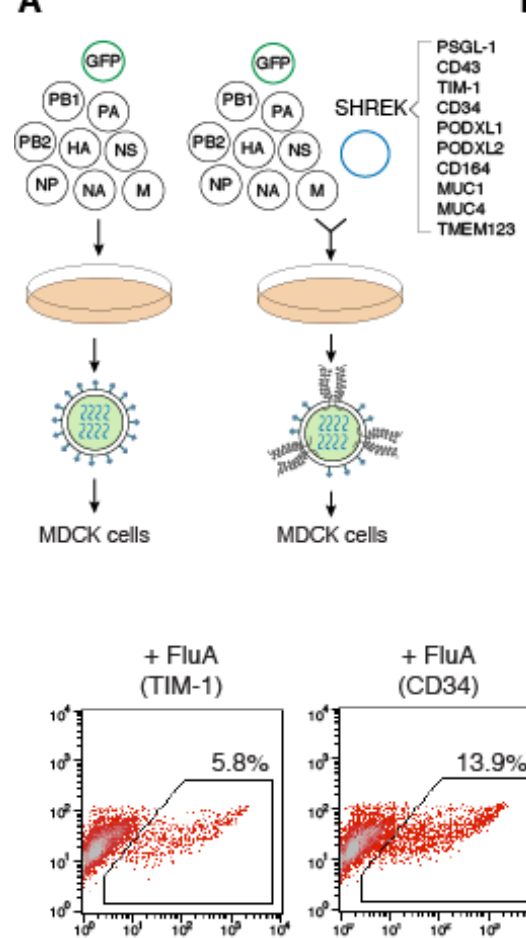

+ FluA

(CD164)
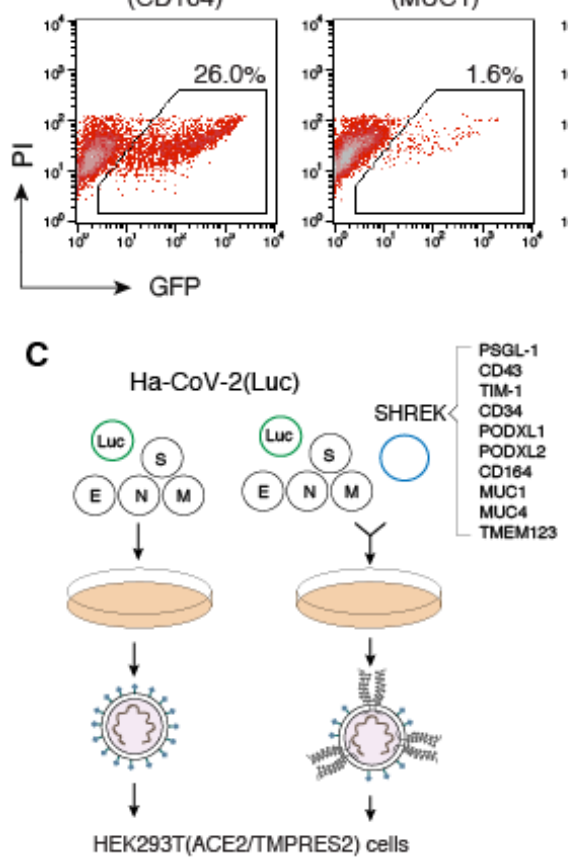

B

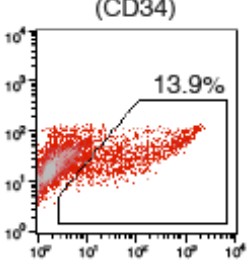

+ FluA

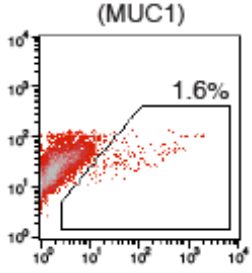

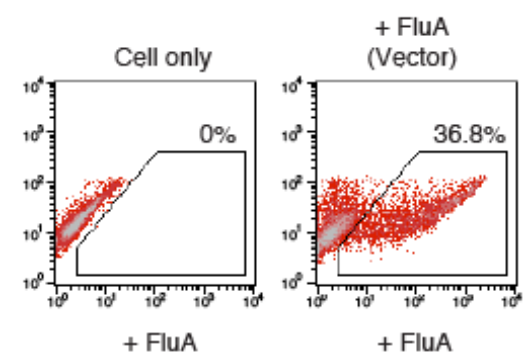
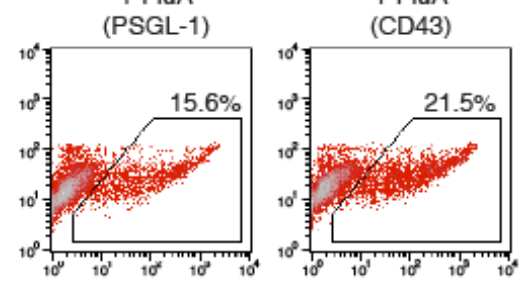

+ FluA

(PDOXL1)

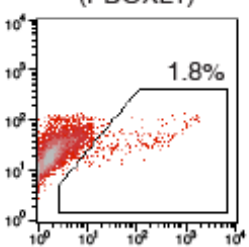

+ FluA

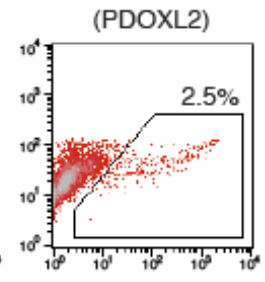

+ FluA

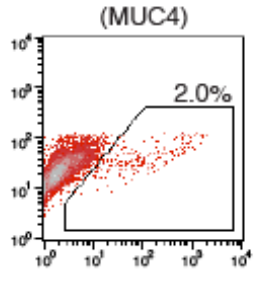

+ FluA

(TMEM123)
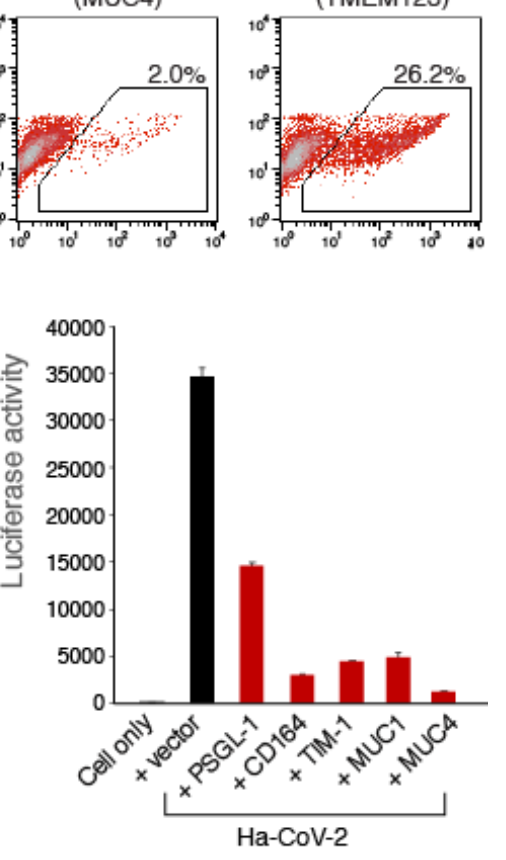

Figure 6. SHREK proteins are broad-spectrum host antiviral factors.

(A) SHREK proteins inactivate influenza A virion infectivity. Influenza A-GFP reporter viruses were assembled by cotransfection of HEK293T cells with eight vectors expressing each of the segments of influenza A/WSN/33 (H1N1) (0.25 $\mu \mathrm{g}$ each), pHW-NA-GFP ( $\triangle \mathrm{AT} 6)(1.5 \mu \mathrm{g})$, and each individual 
bioRxiv preprint doi: https://doi.org/10.1101/2021.02.02.429469; this version posted February 3, 2021. The copyright holder for this preprint (which was not certified by peer review) is the author/funder. All rights reserved. No reuse allowed without permission.

SHREK-expressing vector or an empty vector $(0.5 \mu \mathrm{g})$. Viral particles were harvested at 48 hours and used to infect target MDCK cells. (B) GFP+ cells were quantified by flow cytometry following infection for 24 hours. (C) SHREK proteins inactivate $\mathrm{Ha}-\mathrm{CoV}-2$ virion infectivity. Ha-CoV-2(Luc) particles were assembled in HEK293T cells by cotransfection of SARS-CoV-2 S-, M-, N- and E- expressing vectors (0.3 $\mu \mathrm{g}$ each), Ha-CoV-2(Luc) vector $(1.2 \mu \mathrm{g})$, and each individual SHREK-expressing vector or an empty vector $(1.6 \mu \mathrm{g})$. Virions were harvested, and used to infect HEK293T(ACE2/TMPRSS2) target cells. Luciferase activity was quantified at 18 hours post infection. Data are presented as means \pm SD from triplicate assays. 


\section{MATERIALS AND METHODS}

\section{Cells and cell culture}

HEK293T (ATCC), MDCK (ATCC), Calu-3 (ATCC), and HeLaJC.53 (kindly provided by Dr. David Kabat) cells were maintained in Dulbecco's modified Eagle's medium (DMEM) (Invitrogen) containing 10\% heat-inactivated FBS and 1x penicillin-streptomycin (Invitrogen). HEK293T(ACE2/TMPRSS2) cells (kindly provided by Virongy LLC) were maintained in DMEM (Invitrogen) supplemented with penicillin-streptomycin, puromycin and hygromycin B as instructed by the manufacturer. HIV Rev-dependent GFP indicator Rev-A3R5-GFP cells (Virongy) were cultured in RPMI-1640 plus $10 \%$ FBS supplemented with $1 \mu \mathrm{g} / \mathrm{ml}$ geneticin (G418) (Sigma-Aldrich) and $1 \mu \mathrm{g} / \mathrm{ml}$ puromycin (Sigma-Aldrich).

\section{Plasmid transfection and virus production}

The infectious HIV-1 molecular clone pHIV-1(NL4-3) was obtained from the NIH AIDS Reagent Program. pCMV3-PSGL-1, pCMV3-CD43, pCMV3-CD164, pCMV3-TMEM123, pCMV3-CD34, pCMV3-PODXL1, pCMV3-PODXL2, pCMV3-TIM1, pCMV3-MUC1, pCMV3-MUC4, pCMV3-Empty, and SARS-CoV-2 S, M, E, or N expression vectors were purchased from Sinobiological. The Ha-CoV-2(Luc) vector was synthesized by Twist Bioscience. pCMV6-XL5-ICAM1, pCMV6-XL5-CD2, pCMV6-AC-ITGB2, pCMV6-XL5CD62L were purchased from Origene. The pHW-NA-GFP ( $\triangle \mathrm{AT} 6)$ Reporter plasmid and the A/WSN/1933 H1N1-derived plasmids pHW2000-PB2, pHW2000-PB1, pHW2000-PA, pHW2000-HA, pHW2000-NP, pHW2000-NA, pHW2000-M, and pHW2000-NS were kindly provided by Dr. Feng Li. For HIV-1 viral production, HEK293T cells were cotransfected in a 6well plate with $1 \mu \mathrm{g}$ of pHIV-1(NL4-3) plus the indicated doses of pCMV3-CD164, pCMV3TMEM123, pCMV3-CD34, pCMV3-PODXL1, pCMV3-PODXL2, pCMV3-TIM1, pCMV3MUC1, pCMV3-MUC4 and pCMV3-Empty. Supernatants were collected at 48 hours post transfection. For influenza A-GFP reporter particle assembly, HEK293T cells were cotransfected with pHW2000-PB2, pHW2000-PB1, pHW2000-PA, pHW2000-HA, pHW2000-NP, pHW2000NA, pHW2000-M, and pHW2000-NS (0.25 $\mu \mathrm{g}$ each); pHW-NA-GFP ( $\Delta$ AT6) $(1.5 \mu \mathrm{g})$; and pCMV3-PSGL-1, pCMV3-CD43, pCMV3-CD164, pCMV3-TMEM123, pCMV3-CD34, pCMV3-PODXL1, pCMV3-PODXL2, pCMV3-TIM1, pCMV3-MUC1, pCMV3-MUC4, or pCMV3-Empty DNA (0.5 $\mu \mathrm{g}$ each) in a 6-well plate. Viral supernatants were harvested at 48 hours. Hybrid alphavirus-SARS-CoV-2 (Ha-CoV-2) particles were produced by cotransfection of HEK293T cells in 6-well plates with SARS-CoV-2 S, M, N, and E expression vectors $(0.3 \mu \mathrm{g}$ each), Ha-CoV-2(Luc) (1.2 $\mu \mathrm{g})$, and each individual SHREK-expressing vector or an control empty vector $(1.6 \mu \mathrm{g})$. Ha-CoV-2 particles were harvested at $48 \mathrm{~h}$ post-transfection.

\section{Virus infectivity assays}

For the HIV-1 infectivity assay, p24-normalized HIV-1 particles produced in the presence of PSGL-1, CD43, CD164, TMEM123, CD34, PODXL1, PODXL2, TIM1, MUC1, MUC4, or empty vector were used to infect Rev-A3R5-GFP cells ( 0.2 to 0.5 million cells per infection). The percentage of GFP+ cells was quantified by flow cytometry at 48 or 72 hours post infection. The IC50 inhibition curves were generated using GraphPad Software, La Jolla, California, USA. For the influenza A virion infectivity assay, influenza A-GFP reporter viruses were assembled in the presence of PSGL-1, CD43, CD164, TMEM123, CD34, PODXL1, PODXL2, TIM1, MUC1, MUC4, or empty vectors. Virions were harvested at 48 hours and used to infect MDCK cells $(3 \mathrm{x}$ 
$10^{4}$ cells per infection). GFP expression was quantified at 24 hours post infection by flow cytometry. For Ha-CoV-2 infectivity assay, HEK293T(ACE2/TMPRSS2) cells were infected for 2 hours with Ha-CoV-2 particles assembled in the presence of each individual SHREKexpressing vector or an empty vector. Infected cells were washed and cultured for 18 hours. Cells were lysed with Luciferase Assay Lysis Buffer (Promega). Luminescence was measured on GloMax ${ }^{\circledR}$ Discover Microplate Reader (Promega).

\section{HIV Env incorporation Assay}

HIV-1 particles were produced by co-transfection of HEK293T cells with HIV-1(NL4-3) DNA $(1 \mu \mathrm{g})$ plus $100 \mathrm{ng}$ of vector expressing CD43, CD164, TMEM123, CD34, PODXL1, or PODXL2, or $200 \mathrm{ng}$ of vector expressing MUC-1 or MUC-4. Empty vector was used to keep the amount of DNA equal in each cotransfection. Virus particles were harvested at 48 hours and purified through $10 \%$ sucrose gradient by ultracentrifugation $\left(10,000 \mathrm{x}\right.$ g for 4 hours at $\left.4{ }^{\circ} \mathrm{C}\right)$. Particles were resuspended in LDS lysis buffer (Invitrogen) and subjected to western blot analysis.

\section{Detection of SHREK proteins in HIV-1 particles}

HIV-1 particles were assembled in 6-well plates by co-transfection of HEK293T cells with HIV-1(NL4-3) DNA (1 $\mu \mathrm{g})$ plus an empty vector or the vector expressing each individual SHREK protein (400 ng). Particles were harvested at 48 hours, normalized for HIV-1 p24, and subjected to immuno-magnetic capture as previously described (Thibault et al., 2007). Briefly, magnetic Dynabeads Pan Mouse IgG (Invitrogen) (1x10 8 beads / $0.25 \mathrm{ml})$ were conjugated with one of the following antibodies, mouse anti-PSGL-1 Antibody (KPL-1) (BD Pharmingen), mouse anti-CD43 Antibody (1G10) (BD Biosciences), mouse anti-CD164 Antibody (67D2) (Biolegend), mouse anti-TMEM123 Antibody (297617) (Invitrogen), mouse anti-PODXL1 Antibody (222328) (R \& D Systems), mouse anti-PODXL2 Antibody (211816) (R \& D Systems) or mouse anti-CD34 Antibody (563) (BD Biosciences) for 30 minutes at room temperature. After conjugation, antibody-conjugated beads were incubated with viral particles (an equal p24 level) for $1 \mathrm{hr}$ at $37^{\circ} \mathrm{C}$. The complex was pulled down with a magnet, and washed cold PBS 5 times. Captured viral particles were eluted in 10\% Triton $\mathrm{x}-100$ PBS and quantified by p24 ELISA.

\section{Viral attachment assay}

HIV-1 virus particles produced in the presence of PSGL-1, CD43, CD164, TMEM123, CD34, PODXL1, PODXL2, TIM-1, MUC1, MUC4, or empty vector were incubated with HelaJC.53 cells (prechilled at $4^{\circ} \mathrm{C}$ for $1 \mathrm{~h}$ ) at $4^{\circ} \mathrm{C}$ for $2 \mathrm{~h}$. The cells were then washed extensively (5 times) with cold PBS buffer and then lysed with LDS lysis buffer (Invitrogen) for analysis by Western blot. The attachment of HIV-1 virus produced in the presence of TMEM123 was also separately tested by pre-incubating the virus with mouse monoclonal anti-TMEM123 antibody (297617) (ThermoFisher) $(25 \mathrm{ug} / \mathrm{ml})$ at $37^{\circ} \mathrm{C}$ for $1 \mathrm{~h}$, followed by incubation of the virus with HeLaJC.53 cells at $4^{\circ} \mathrm{C}$ for $2 \mathrm{~h}$ and lysing with LDS buffer.

\section{Western blots}

Cells were lysed in LDS lysis buffer (Invitrogen). Proteins were denatured by boiling in sample buffer and subjected to SDS-PAGE, transferred to nitrocellulose membrane, and incubated overnight at $4{ }^{\circ} \mathrm{C}$ with one of the following primary antibodies: mouse anti-HIV-1 p24 monoclonal antibody (183-H12-5C) (NIH AIDS Reagent Program) (1:1000 dilution), human 
anti-HIV-1 gp41 (2F5) (1:1000 dilution) (NIH AIDS Reagent Program), mouse antiMUC1antibody (HMPV) (BD Biosciences) (1:1000 dilution), mouse monoclonal anti-MUC4 antibody (1G8) (ThermoFisher) (1:1000 dilution), mouse monoclonal anti-TMEM123 antibody (297617) (ThermoFisher) (1:1000 dilution), or anti-GAPDH goat polyclonal antibody (Abcam) (1:1000 dilution). Membranes were incubated with HRP-labeled goat anti-mouse IgG (KPL) (1:2500 dilution) for $60 \mathrm{~min}$ at room temperature, or goat anti-human $800 \mathrm{cw}$-labeled antibodies (Li-Cor Biosciences) (1:2500 dilution) for $30 \mathrm{~min}$ at room temperature. Chemiluminescence signal was detected by using West Femto chemiluminescence substrate (Thermo Fisher Scientific), and images were captured with a CCD camera (FluorChem 9900 Imaging Systems) (Alpha Innotech). Blots stained with infrared antibodies were scanned with an Odyssey infrared imager (Li-Cor Biosciences).

\section{p24 ELISA}

Detection of extracellular HIV-1 p24 were performed using an in-house p24 ELISA kit. Briefly, microtiter plates (Sigma-Aldrich) were coated with anti-HIV-1 p24 monoclonal antibody (183-H12-5C) (NIH AIDS Reagent Program). Samples were incubated for 2 hours at $37^{\circ} \mathrm{C}$, followed by washing and incubating with biotinylated anti-HIV immune globulin (HIVIG) (NIH AIDS Reagent Program) for 1 hour at $37^{\circ} \mathrm{C}$. Plates were then washed and incubated with avidinperoxidase conjugate ( $\mathrm{R} \& \mathrm{D}$ Systems) for 1 hour at $37^{\circ} \mathrm{C}$, followed by washing and incubating with tetramethylbenzidine (TMB) substrate. Plates were kinetically read using an ELx808 automatic microplate reader (Bio-Tek Instruments) at $630 \mathrm{~nm}$.

\section{Surface staining}

HEK293T cells were transfected with 400 ng of pCMV3-PSGL-1, pCMV3-CD43, pCMV3CD164, pCMV3-CD34, pCMV3-PODXL1, pCMV3-PODXL2, pCMV3-TIM1, or pCMV3Empty DNA. At 48 hours post transfection, 0.5-1 million cells were stained with one of the following primary antibodies: mouse anti-PSGL1 antibody (KPL-1) (BD Pharmingen), mouse anti-CD43 antibody (1G10) (BD Biosciences), mouse anti-CD164 antibody (67D2) (Biolegend), mouse anti-CD34 antibody (563) (BD Biosciences), mouse anti-TIM1 antibody (219211) (R \& D Systems), mouse monoclonal anti-PODXL1 antibody (222328) (R \& D systems), or mouse monoclonal anti-PODXL2 antibody (211816) (R \& D Systems), followed by staining with Alexa Fluor 488-conjugated goat anti-mouse secondary antibody (Invitrogen). 


\section{REFERENCES}

Baisse, B., Galisson, F., Giraud, S., Schapira, M., and Spertini, O. (2007). Evolutionary conservation of P-selectin glycoprotein ligand-1 primary structure and function. BMC Evol Biol 7, 166.

Barclay, A.N. (2003). Membrane proteins with immunoglobulin-like domains - a master superfamily of interaction molecules. Seminars in Immunology 15, 215-223.

Carter, C.C., Onafuwa-Nuga, A., McNamara, L.A., Riddell, J.t., Bixby, D., Savona, M.R., and Collins, K.L. (2010). HIV-1 infects multipotent progenitor cells causing cell death and establishing latent cellular reservoirs. Nat Med 16, 446-451.

Doyonnas, R., Nielsen, J.S., Chelliah, S., Drew, E., Hara, T., Miyajima, A., and McNagny, K.M. (2005). Podocalyxin is a CD34-related marker of murine hematopoietic stem cells and embryonic erythroid cells. Blood 105, 4170-4178.

Freed, E.O. (2015). HIV-1 assembly, release and maturation. Nat Rev Microbiol 13, 484-496.

Fu, Y., He, S., Waheed, A.A., Dabbagh, D., Zhou, Z., Trinite, B., Wang, Z., Yu, J., Wang, D., Li, F., et al. (2020). PSGL-1 restricts HIV-1 infectivity by blocking virus particle attachment to target cells. Proc Natl Acad Sci U S A 117, 9537-9545.

Goetz, D.J., Greif, D.M., Ding, H., Camphausen, R.T., Howes, S., Comess, K.M., Snapp, K.R., Kansas, G.S., and Luscinskas, F.W. (1997). Isolated P-selectin glycoprotein ligand-1 dynamic adhesion to P- and E-selectin. J Cell Biol 137, 509-519.

Guyer, D.A., Moore, K.L., Lynam, E.B., Schammel, C.M., Rogelj, S., McEver, R.P., and Sklar, L.A. (1996). P-selectin glycoprotein ligand-1 (PSGL-1) is a ligand for L-selectin in neutrophil aggregation. Blood 88, 2415-2421.

Habte, H.H., Kotwal, G.J., Lotz, Z.E., Tyler, M.G., Abrahams, M., Rodriques, J., Kahn, D., and Mall, A.S. (2007). Antiviral activity of purified human breast milk mucin. Neonatology 92, 96104.

Hattrup, C.L., and Gendler, S.J. (2008). Structure and function of the cell surface (tethered) mucins. Annu Rev Physiol 70, 431-457.

He, S., Waheed, A.A., Hetrick, B., Dabbagh, D., Akhrymuk, I.V., Kehn-Hall, K., Freed, E.O., and $\mathrm{Wu}, \mathrm{Y}$. (2021). PSGL-1 Inhibits the Incorporation of SARS-CoV and SARS-CoV-2 Spike Glycoproteins into Pseudovirions and Impairs Pseudovirus Attachment and Infectivity Viruses 13,46 .

Hetrick, B., He, S., Chilin, L.D., Dabbagh, D., Alem, F., Narayanan, A., Luchini, A., Li, T., Liu, X., Liotta, L., et al. (2020). Development of a novel hybrid alphavirus-SARS-CoV-2 particle for rapid in vitro screening and quantification of neutralization antibodies, antiviral drugs, and viral mutations. bioRxiv, 2020.2012.2022.423965.

Hynes, R.O. (2002). Integrins: Bidirectional, Allosteric Signaling Machines. Cell 110, 673-687.

Ley, K. (2003). The role of selectins in inflammation and disease. Trends in Molecular Medicine 9, 263-268. 
Li, F., Erickson, H.P., James, J.A., Moore, K.L., Cummings, R.D., and McEver, R.P. (1996a). Visualization of P-selectin glycoprotein ligand-1 as a highly extended molecule and mapping of protein epitopes for monoclonal antibodies. J Biol Chem 271, 6342-6348.

Li, F., Wilkins, P.P., Crawley, S., Weinstein, J., Cummings, R.D., and McEver, R.P. (1996b). Post-translational modifications of recombinant P-selectin glycoprotein ligand-1 required for binding to P- and E-selectin. J Biol Chem 271, 3255-3264.

Li, M., Waheed, A.A., Yu, J., Zeng, C., Chen, H.Y., Zheng, Y.M., Feizpour, A., Reinhard, B.M., Gummuluru, S., Lin, S., et al. (2019). TIM-mediated inhibition of HIV-1 release is antagonized by Nef but potentiated by SERINC proteins. Proc Natl Acad Sci U S A 116, 5705-5714.

Liu, Y., Fu, Y., Wang, Q., Li, M., Zhou, Z., Dabbagh, D., Fu, C., Zhang, H., Li, S., Zhang, T., et al. (2019). Proteomic profiling of HIV-1 infection of human CD4(+) T cells identifies PSGL-1 as an HIV restriction factor. Nat Microbiol 4, 813-825.

Liu, Y., Song, Y., Zhang, S., Diao, M., Huang, S., Li, S., and Tan, X. (2020). PSGL-1 inhibits HIV-1 infection by restricting actin dynamics and sequestering HIV envelope proteins. Cell Discov 6, 53.

Ma, F., Zhang, C., Prasad, K.V., Freeman, G.J., and Schlossman, S.F. (2001). Molecular cloning of Porimin, a novel cell surface receptor mediating oncotic cell death. Proc Natl Acad Sci U S A 98, 9778-9783.

McEver, R.P., Moore, K.L., and Cummings, R.D. (1995). Leukocyte trafficking mediated by selectin-carbohydrate interactions. J Biol Chem 270, 11025-11028.

McLaren, P.J., Gawanbacht, A., Pyndiah, N., Krapp, C., Hotter, D., Kluge, S.F., Gotz, N., Heilmann, J., Mack, K., Sauter, D., et al. (2015). Identification of potential HIV restriction factors by combining evolutionary genomic signatures with functional analyses. Retrovirology 12,41 .

Murakami, T., Carmona, N., and Ono, A. (2020). Virion-incorporated PSGL-1 and CD43 inhibit both cell-free infection and transinfection of HIV-1 by preventing virus-cell binding. Proc Natl Acad Sci U S A 117, 8055-8063.

Nielsen, J.S., and McNagny, K.M. (2008). Novel functions of the CD34 family. J Cell Sci 121, 3683-3692.

Pallant, A., Eskenazi, A., Mattei, M.G., Fournier, R.E., Carlsson, S.R., Fukuda, M., and Frelinger, J.G. (1989). Characterization of cDNAs encoding human leukosialin and localization of the leukosialin gene to chromosome 16. Proc Natl Acad Sci U S A 86, 1328-1332.

Patel, K.D., Nollert, M.U., and McEver, R.P. (1995). P-selectin must extend a sufficient length from the plasma membrane to mediate rolling of neutrophils. J Cell Biol 131, 1893-1902.

Plante, J.A., Plante, K.S., Gralinski, L.E., Beall, A., Ferris, M.T., Bottomly, D., Green, R., McWeeney, S.K., Heise, M.T., Baric, R.S., et al. (2020). Mucin 4 Protects Female Mice from Coronavirus Pathogenesis. bioRxiv 2020.02.19.957118.

Sako, D., Chang, X.J., Barone, K.M., Vachino, G., White, H.M., Shaw, G., Veldman, G.M., Bean, K.M., Ahern, T.J., Furie, B., et al. (1993). Expression cloning of a functional glycoprotein ligand for P-selectin. Cell 75, 1179-1186. 
Sassetti, C., Van Zante, A., and Rosen, S.D. (2000). Identification of endoglycan, a member of the CD34/podocalyxin family of sialomucins. J Biol Chem 275, 9001-9010.

Sidney, L.E., Branch, M.J., Dunphy, S.E., Dua, H.S., and Hopkinson, A. (2014). Concise review: evidence for CD34 as a common marker for diverse progenitors. Stem Cells 32, 1380-1389.

Tauxe, C., Xie, X., Joffraud, M., Martinez, M., Schapira, M., and Spertini, O. (2008). P-selectin glycoprotein ligand-1 decameric repeats regulate selectin-dependent rolling under flow conditions. J Biol Chem 283, 28536-28545.

Thibault, S., Tardif, M.R., Gilbert, C., and Tremblay, M.J. (2007). Virus-associated host CD62L increases attachment of human immunodeficiency virus type 1 to endothelial cells and enhances trans infection of CD4+ T lymphocytes. J Gen Virol 88, 2568-2573.

Umetsu, S.E., Lee, W.L., McIntire, J.J., Downey, L., Sanjanwala, B., Akbari, O., Berry, G.J., Nagumo, H., Freeman, G.J., Umetsu, D.T., et al. (2005). TIM-1 induces T cell activation and inhibits the development of peripheral tolerance. Nat Immunol 6, 447-454.

V'kovski, P., Kratzel, A., Steiner, S., Stalder, H., and Thiel, V. (2020). Coronavirus biology and replication: implications for SARS-CoV-2. Nature Reviews Microbiology.

Watt, S.M., and Chan, J.Y. (2000). CD164--a novel sialomucin on CD34+ cells. Leuk Lymphoma 37, 1-25.

Wu, Y., Beddall, M.H., and Marsh, J.W. (2007a). Rev-dependent indicator T cell line. Current HIV Research 5, 395-403.

Wu, Y., Beddall, M.H., and Marsh, J.W. (2007b). Rev-dependent lentiviral expression vector. Retrovirology 4, 12. 


\section{SUPPLEMENTARY FIGURES}
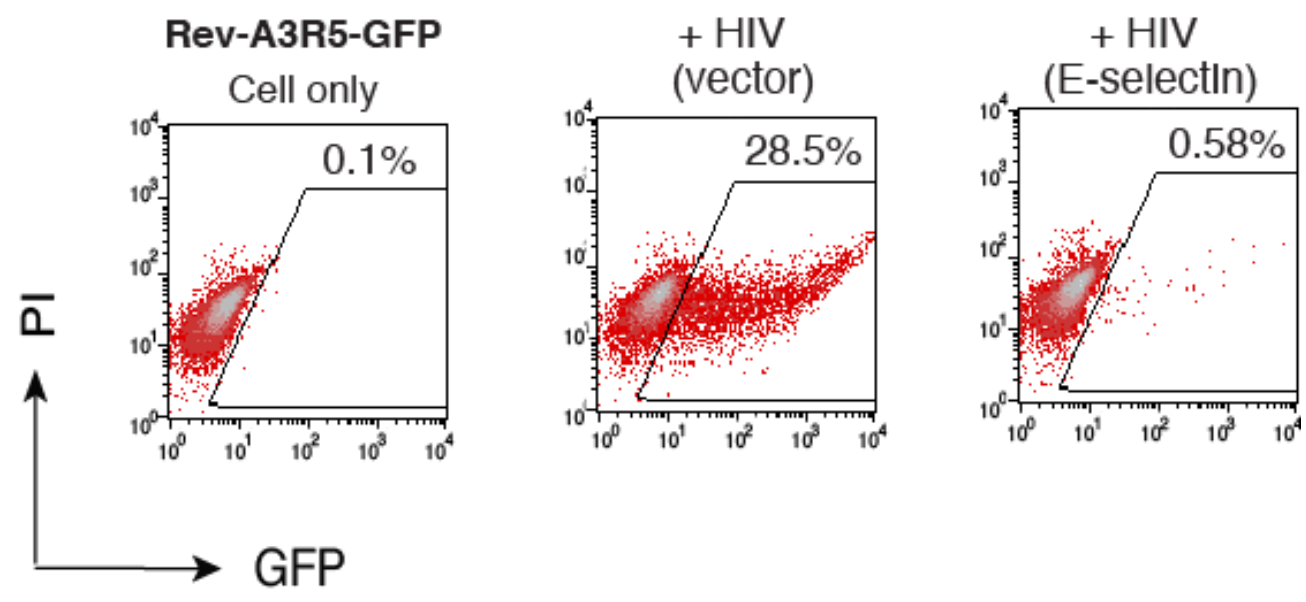

Figure 1 Supplement 1. E-selectin inactivates HIV-1 virion infectivity.

HEK293T cells were cotransfected with HIV(NL4-3) DNA (1 $\mu \mathrm{g})$ plus the vector expressing Eselectin or an empty vector (400 ng). Virions were harvested at 48 hours and normalized for p24, and viral infectivity was quantified by infecting Rev-A3R5-GFP indicator cells. Shown are the percentages of GFP + cells at 72 hours post infection. 

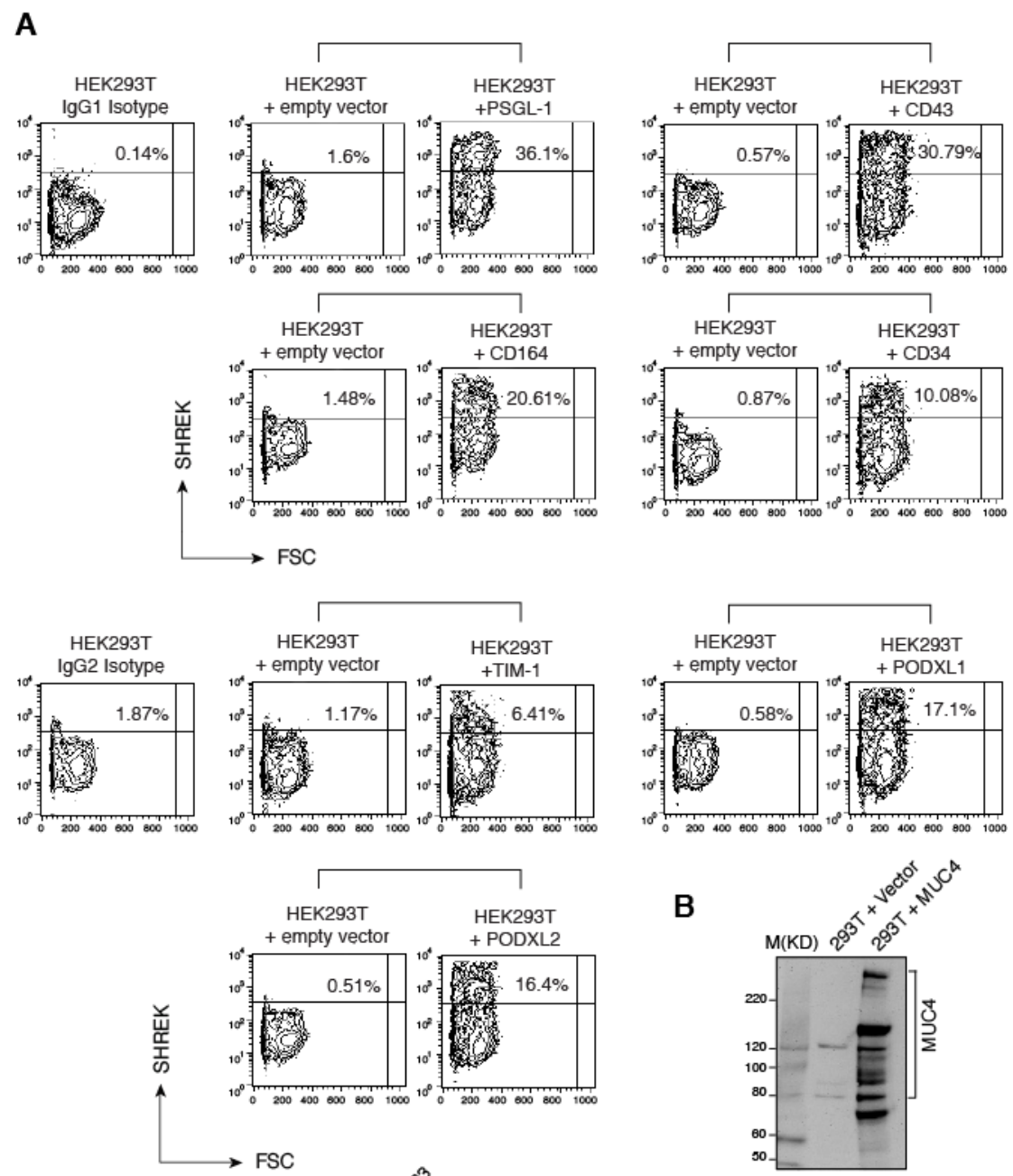

B
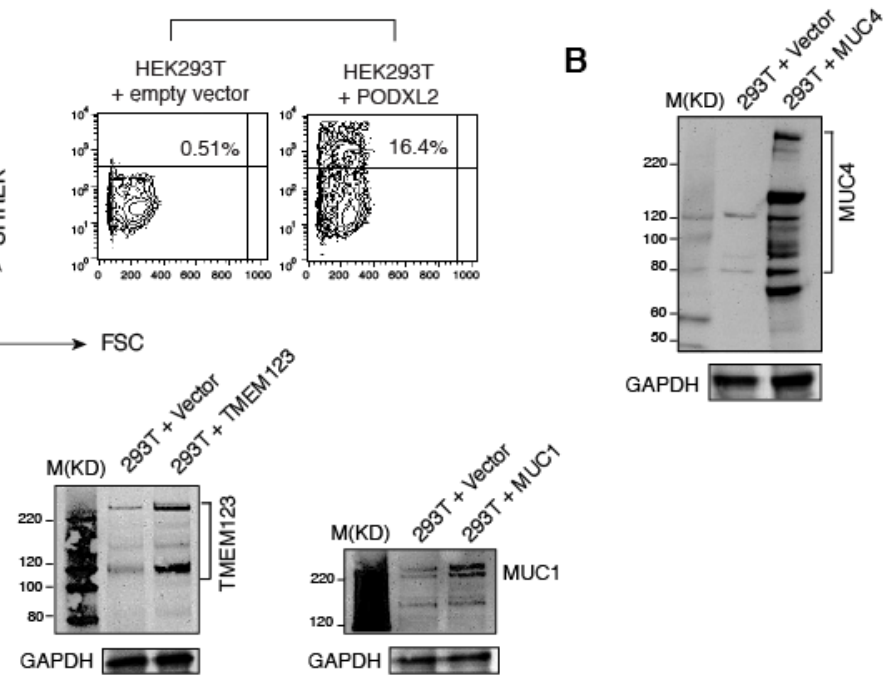

Figure 1 Supplement 2. Expression of SHREK proteins in HEK293T cells.

(A) HEK293T cells were transfected with 400 ng of either PSGL-1, CD43, CD164, CD34, TIM1, PODXL1, or PODXL2. Expression of each protein was quantified at 48 hours by surface staining using antibodies against each of the indicated proteins. (B) HEK293T cells were transfected with $400 \mathrm{ng}$ of MUC1, MUC4, TMEM123, or an empty vector. Expression of each protein was measured by Western blot using antibodies against MUC1, MUC4 or TMEM123. 

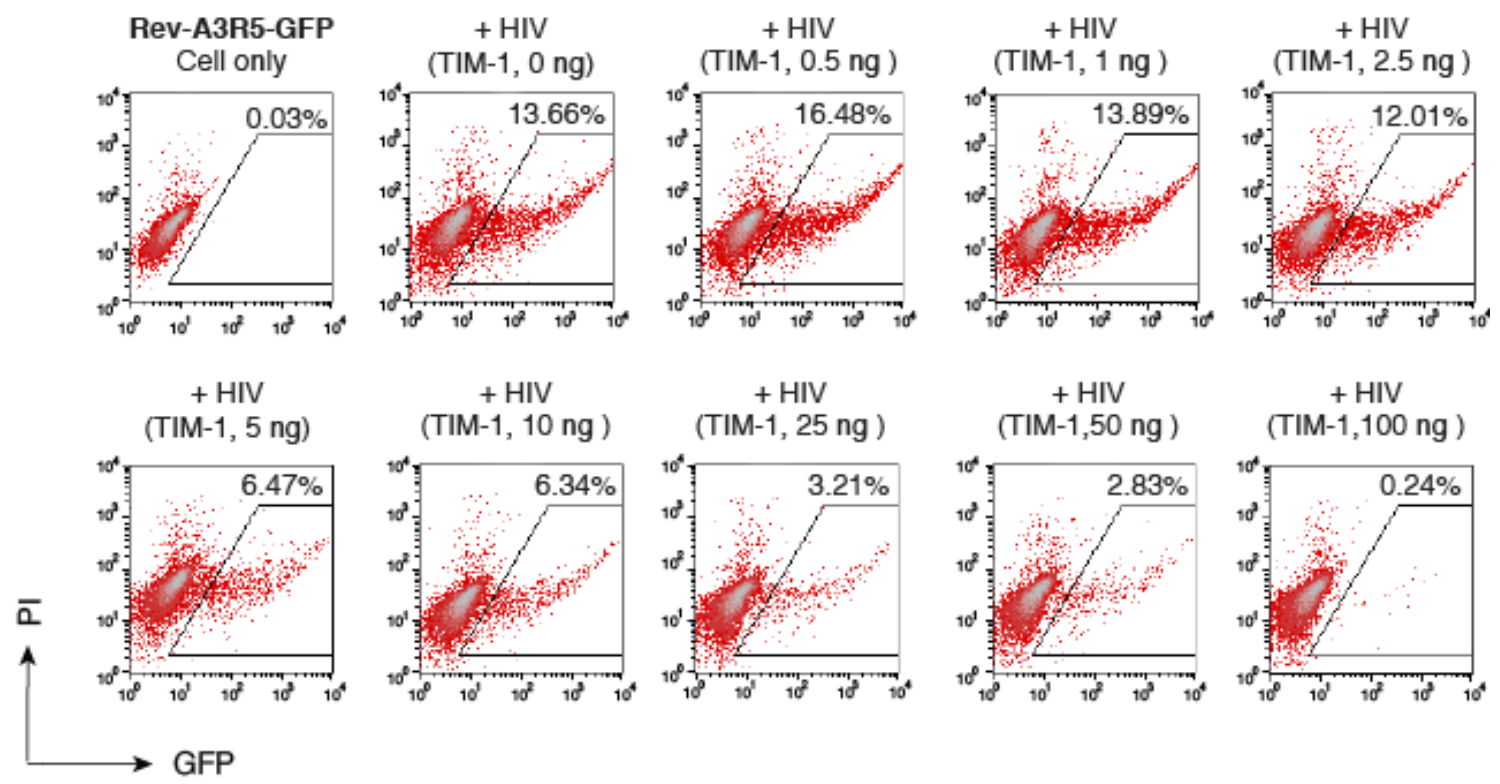

Figure 2 Supplement 1. Dose-dependent inhibition of HIV-1 by TIM-1.

HIV-1 particles were produced in HEK293T cells by co-transfection of NL4-3 DNA (1 $\mu$ g) plus an empty vector or the indicated doses of TIM-1. P24-normalized virions were used to infect Rev-A3R5-GFP cells. The percentage of GFP + cells was quantified by flow cytometry at 72 hours post infection. 


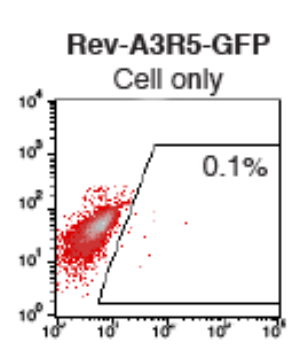

+ HIV

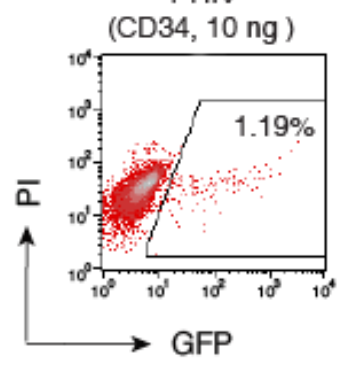

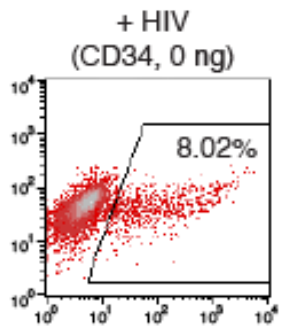

+ HIV

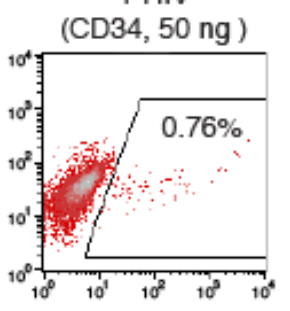

+ HIV

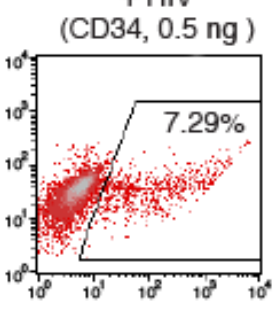

+ HIV

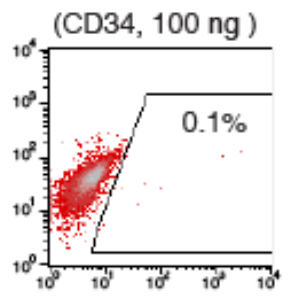

+ HIV

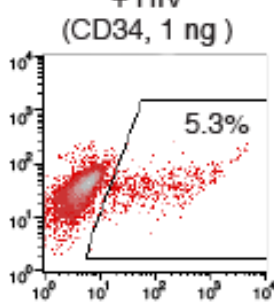

+ HIV
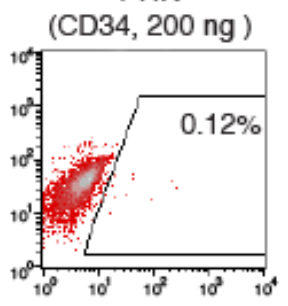

+ HIV

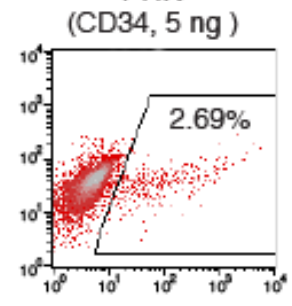

+ HIV

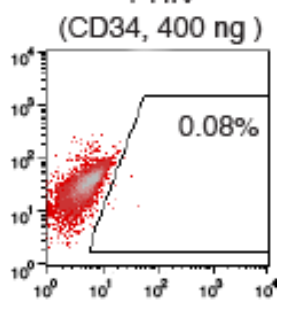

\section{Figure 2 Supplement 2. Dose-dependent inhibition of HIV-1 by CD34.}

HIV-1 particles were produced in HEK293T cells by co-transfection of NL4-3 DNA $(1 \mu \mathrm{g})$ plus an empty vector or the indicated doses of CD34. P24-normalized virions were used to infect Rev-A3R5-GFP cells. The percentage of GFP+ cells was quantified by flow cytometry at 72 hours post infection. 

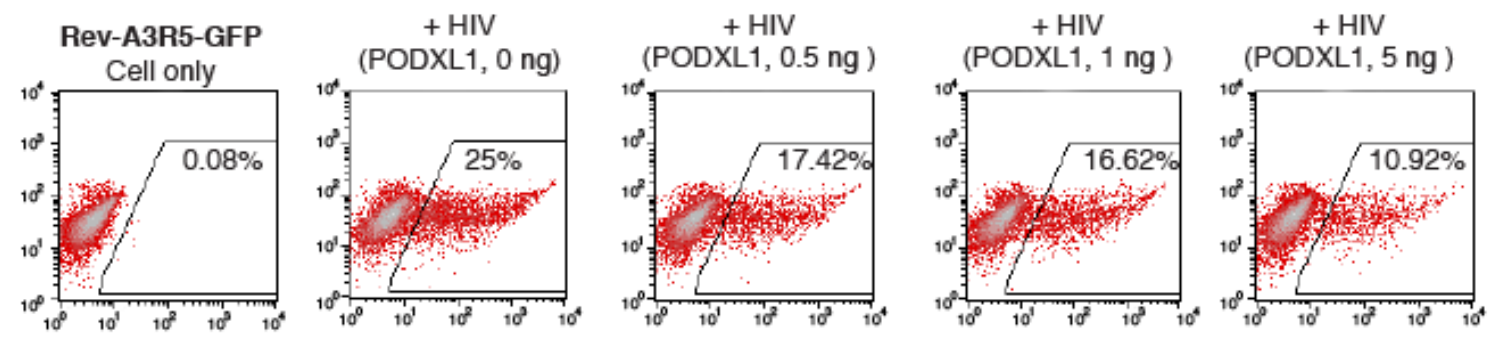

+ HIV

+ HIV

+ HIV

+ HIV

+ HIV
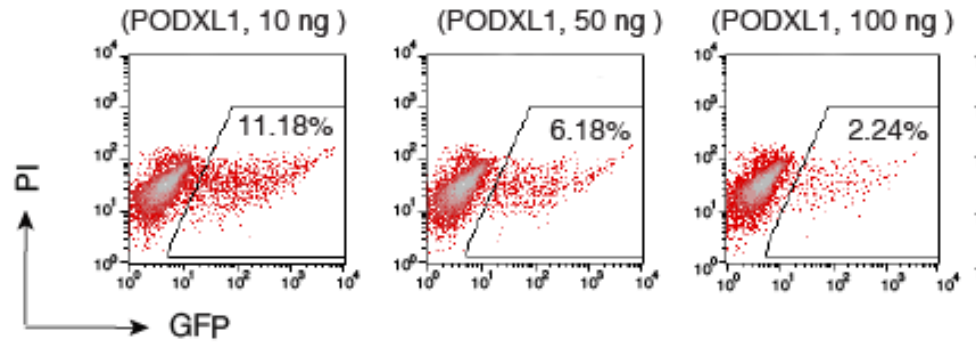

(PODXL1, $200 \mathrm{ng}$ )

(PODXL1, $400 \mathrm{ng}$ )
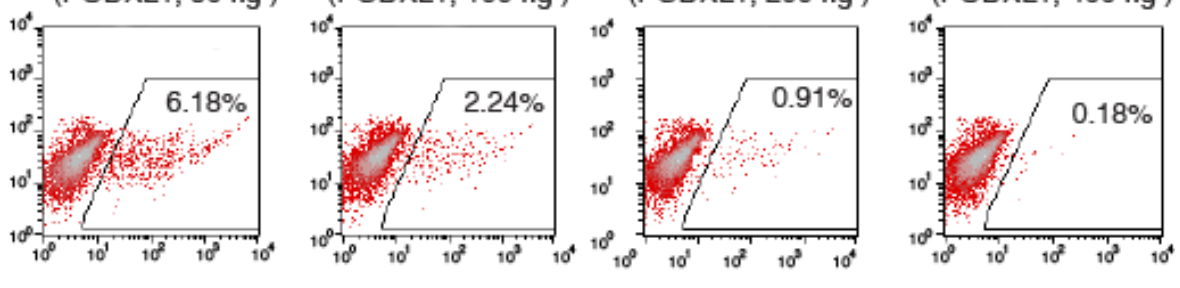

Figure 2 Supplement 3. Dose-dependent inhibition of HIV-1 by PODXL1.

HIV-1 particles were produced in HEK293T cells by co-transfection of NL4-3 DNA $(1 \mu \mathrm{g})$ plus an empty vector or the indicated doses of PODXL1. P24-normalized virions were used to infect Rev-A3R5-GFP cells. The percentage of GFP+ cells was quantified by flow cytometry at 72 hours post infection. 

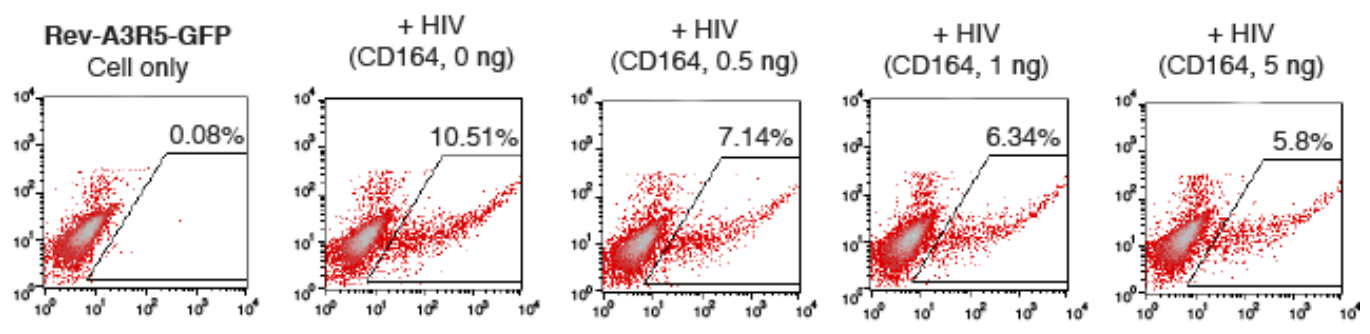

+ HIV (CD164, $10 \mathrm{ng})$

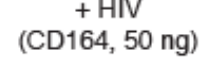

+ HIV

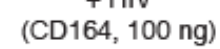

+ HIV
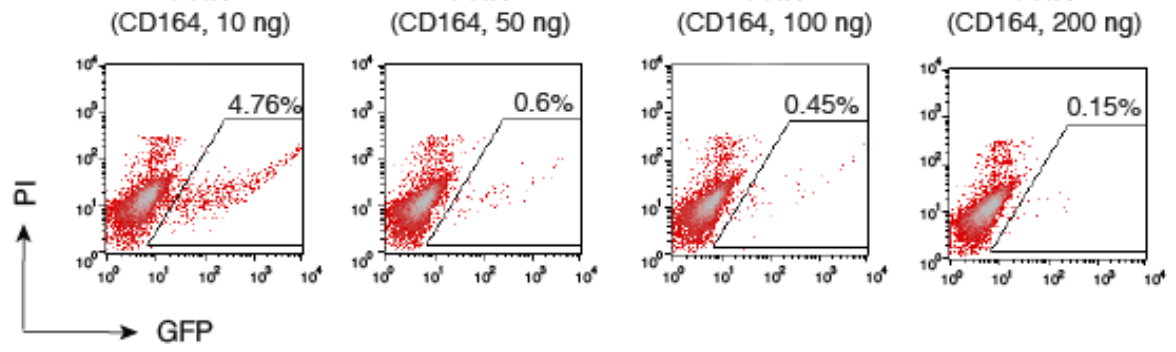

Figure 2 Supplement 4. Dose-dependent inhibition of HIV-1 by CD164.

HIV-1 particles were produced in HEK293T cells by co-transfection of NL4-3 DNA ( $1 \mu \mathrm{g})$ plus an empty vector or the indicated doses of CD164. P24-normalized virions were used to infect Rev-A3R5-GFP cells. The percentage of GFP+ cells was quantified by flow cytometry at 72 hours post infection. 

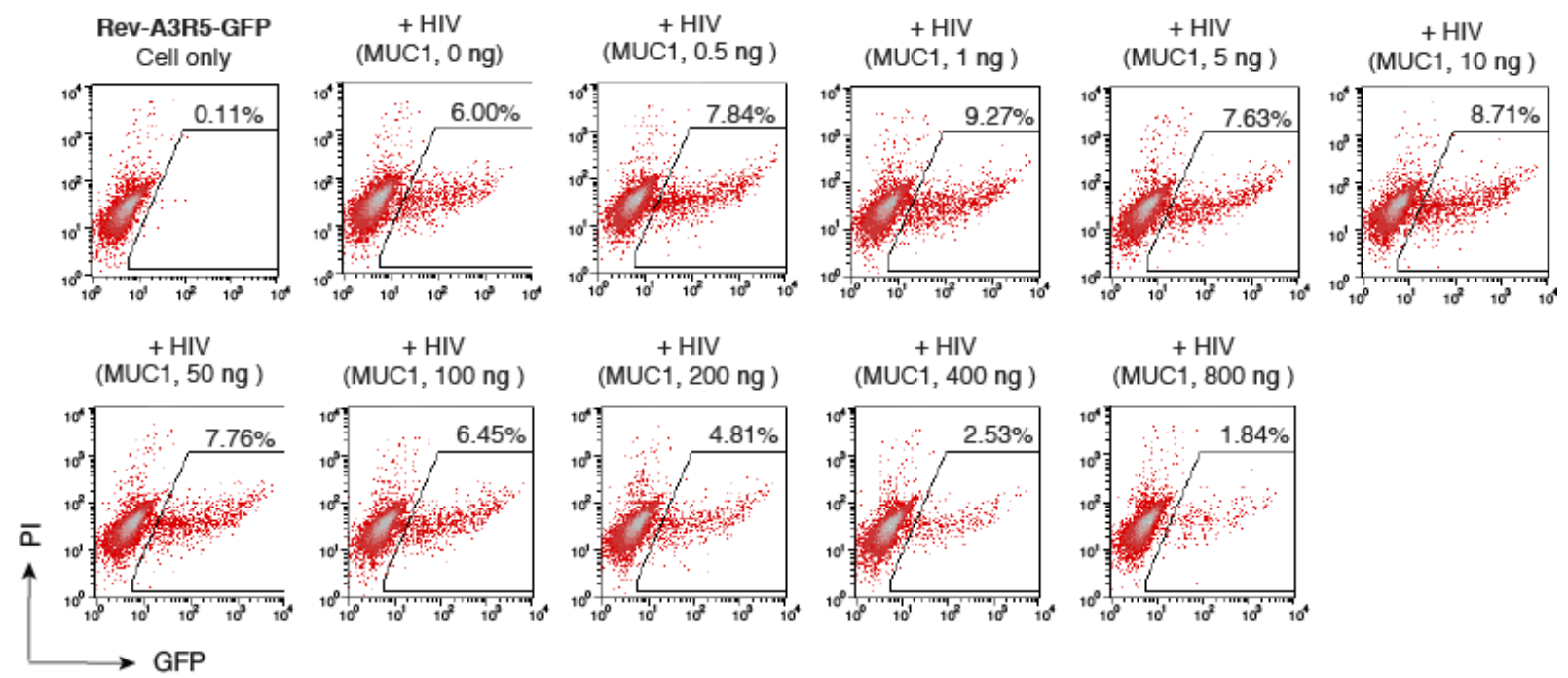

GrP

\section{Figure 2 Supplement 5. Dose-dependent inhibition of HIV-1 by MUC1.}

HIV-1 particles were produced in HEK293T cells by co-transfection of NL4-3 DNA ( $1 \mu \mathrm{g})$ plus an empty vector, or the indicated doses of MUC1. P24-normalized virions were used to infect Rev-A3R5-GFP cells. The percentage of GFP+ cells was quantified by flow cytometry at 72 hours post infection. 

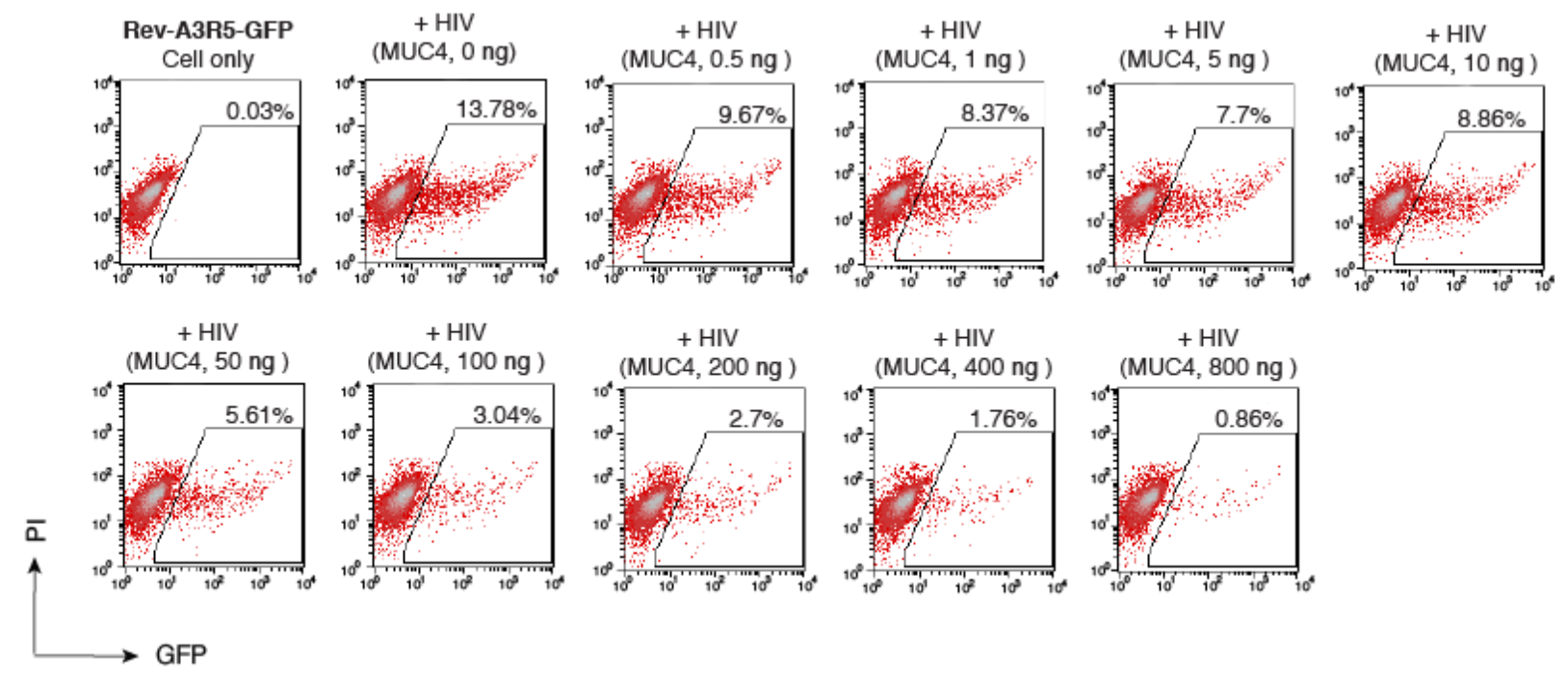

\section{Figure 2 Supplement 6. Dose-dependent inhibition of HIV-1 by MUC4.}

HIV-1 particles were produced in HEK293T cells by co-transfection of NL4-3 DNA ( $1 \mu \mathrm{g})$ plus an empty vector or the indicated doses of MUC4. P24-normalized virions were used to infect Rev-A3R5-GFP cells. The percentage of GFP+ cells was quantified by flow cytometry at 72 hours post infection. 

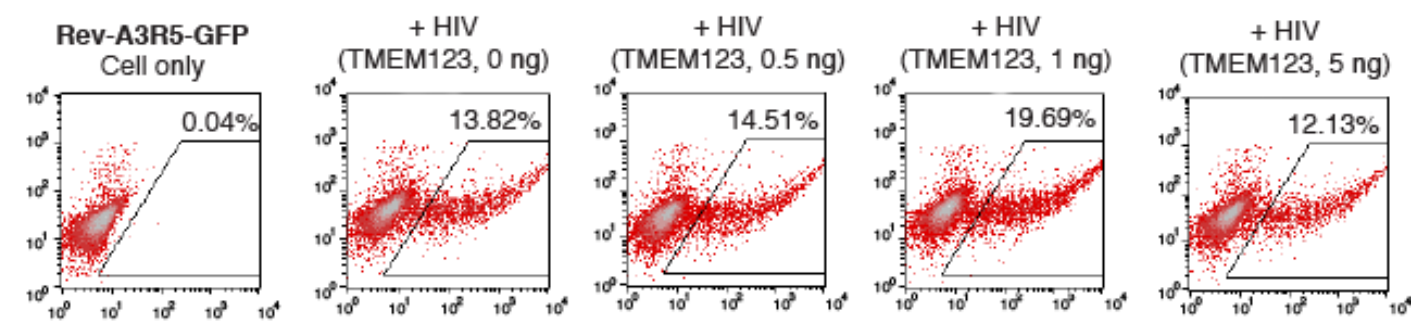

+ HIV

+ HIV

+ HIV

+ HIV

(TMEM123, $10 \mathrm{ng}$ )
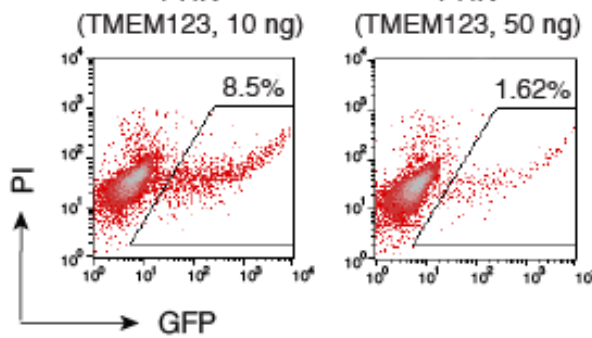

(TMEM123, $100 \mathrm{ng}$ )

(TMEM123, $200 \mathrm{ng}$ )
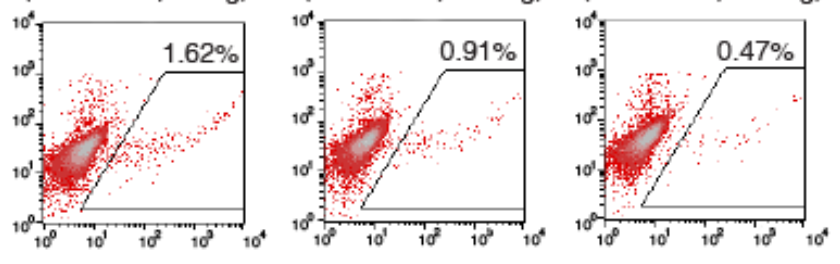

$\rightarrow$ GFP

Figure 2 Supplement 7. Dose-dependent inhibition of HIV-1 by TMEM123.

HIV-1 particles were produced in HEK293T cells by co-transfection of NL4-3 DNA ( $1 \mu \mathrm{g})$ plus an empty vector, or the indicated doses of TMEM234. P24-normalized virions were used to infect Rev-A3R5-GFP cells. The percentage of GFP+ cells was quantified by flow cytometry at 72 hours post infection. 


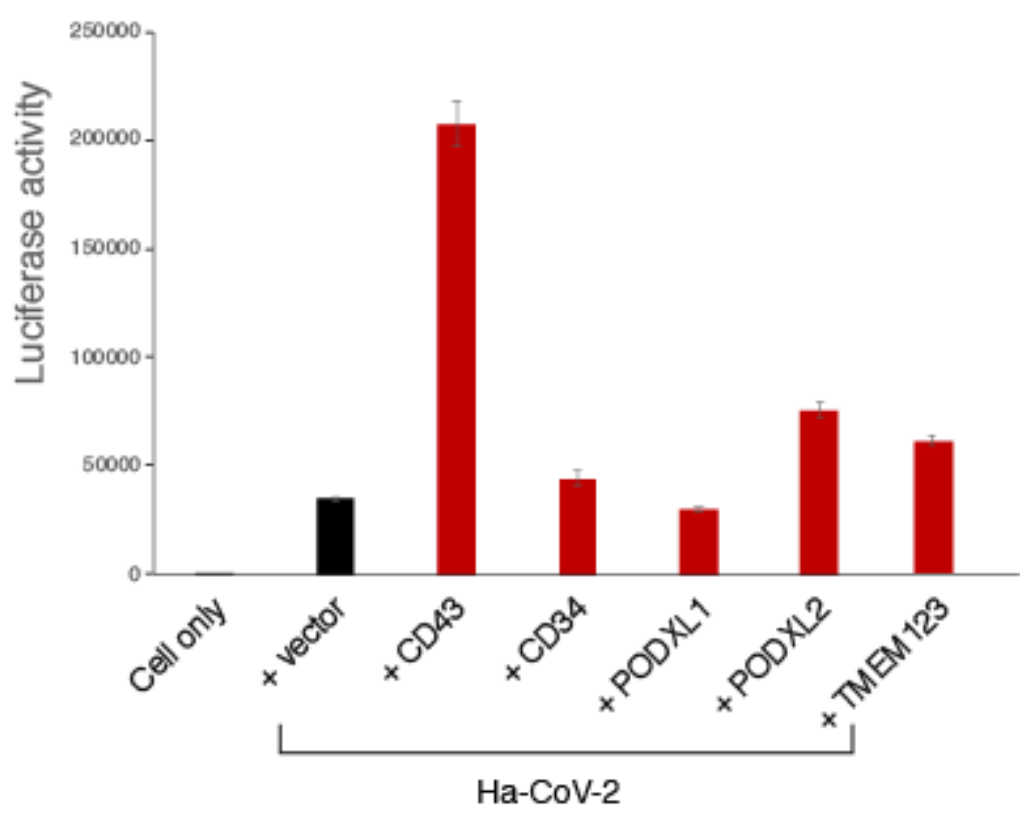

Figure 6 Supplement 1. Lack of inhibition of Ha-CoV-2 virion infectivity by CD43, CD43, PODXL1, PODXL2, and TMEM123.

Ha-CoV-2(Luc) particles were assembled in HEK293T cells by cotransfection of SARS-CoV-2 S-, M-, N- and E- expressing vectors ( $0.3 \mu \mathrm{g}$ each), Ha-CoV-2(Luc) vector $(1.2 \mu \mathrm{g})$, and each individual SHREK-expressing vector (CD43, CD34, PODXL1, PODXL2, or TMEM123) or an empty vector $(1.6 \mu \mathrm{g})$. Virions were harvested, and used to infect HEK293T(ACE2/TMPRSS2) target cells. Luciferase activity was quantified at 18 hours post infection. Data are presented as triplicate means $\pm \mathrm{SD}$. 